\title{
Caspase-11 regulates the tumour suppressor function of STAT1 in a murine model of colitis-associated carcinogenesis
}

\author{
Brian Flood ${ }^{1} \cdot$ Joan Manils $^{1} \cdot$ Ciara Nulty $^{1} \cdot$ Ewelina Flis $^{1} \cdot$ Sinead Kenealy ${ }^{1} \cdot$ Gillian Barber $^{1} \cdot$ Joanna Fay $^{2}$.

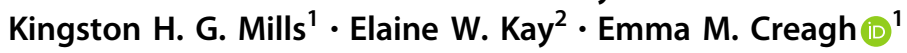

Received: 4 May 2018 / Revised: 13 November 2018 / Accepted: 23 November 2018 / Published online: 11 December 2018

(c) The Author(s) 2018. This article is published with open access

\begin{abstract}
Murine inflammatory caspase-11 has an important role in intestinal epithelial inflammation and barrier function. Activation of the non-canonical inflammasome, mediated by caspase-11, serves as a regulatory pathway for the production of the proinflammatory cytokines IL-1 $\beta$ and IL-18, and has a key role in pyroptotic cell death. We have previously demonstrated a protective role for caspase-11 during dextran sulphate sodium (DSS)-induced colitis, however the importance of caspase-11

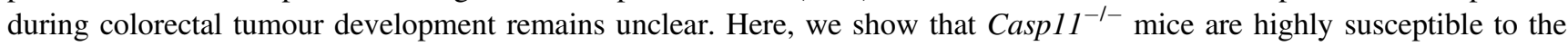
azoxymethane (AOM)-DSS model of colitis-associated cancer (CAC), compared to their wild type (WT) littermates. We show that deficient IL-18 production occurs at initial inflammation stages of disease, and that IL-1 $\beta$ production is more

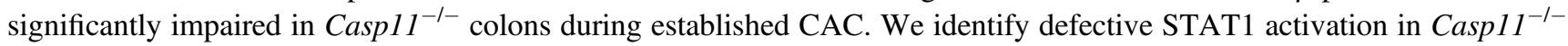
colons during disease progression, and show that IL-1 $\beta$ signalling induces caspase-11 expression and STAT1 activation in primary murine macrophages and intestinal epithelial cells. These findings uncover an anti-tumour role for the caspase-11 and the non-canonical inflammasome during CAC, and suggest a critical role for caspase-11, linking IL-1 $\beta$ and STAT1 signalling pathways.
\end{abstract}

\section{Introduction}

Colorectal cancer (CRC) represents the second most common incident solid organ cancer in both females and males, with an estimated annual incidence in 2008 of 1.2 million worldwide [1]. Innate immunity significantly contributes to acute inflammation, and has a critical role in the pathogenesis of inflammatory bowel diseases (IBDs) [2], which are risk factors for the development of colitis-associated cancer (CAC) [3]. Although not fully understood, the

These authors contributed equally: Brian Flood, Joan Manils.

Supplementary material The online version of this article (https:// doi.org/10.1038/s41388-018-0613-5) contains supplementary material, which is available to authorized users.

Emma M. Creagh

ecreagh@tcd.ie

1 Trinity Biomedical Sciences Institute, School of Biochemistry \& Immunology, Trinity College Dublin, Dublin 2, Ireland

2 Royal College of Surgeons in Ireland and Beaumont Hospital, Dublin 9, Ireland progression of IBD to CAC is associated with persistent immune cell infiltration and inflammatory cytokine production, under the regulation of inflammation-associated transcription factors, such as NFkB and STAT1 [4].

Canonical inflammasome-mediated caspase-1 activation results in the cleavage and secretion of the proinflammatory cytokines IL- $1 \beta$ and IL-18 [5, 6], and is also associated with pyroptosis, the pro-inflammatory form of cell death [7]. The non-canonical inflammasome, mediated by caspase-11, serves as an additional regulatory pathway for caspase- 1 maturation of IL- $1 \beta$ and IL-18 in response to Gram-negative bacteria, such as Escherichia coli and Citrobacter rodentium, which introduce LPS into the host cytoplasm during infection $[8,9]$. Pyroptosis is also inducible by caspase-11, independently of canonical inflammasome assembly, in response to cytosolic LPS $[10,11]$.

Caspase-11 is unique among caspases in that it is highly regulated at levels of transcription upon the activation of inflammatory responses. Pro-caspase-11 is inducible upon stimulation with activators of innate immunity, including LPS, IFN $\gamma$, and type I IFN [12-15]. In the context of intestinal inflammation, we and others have demonstrated a 


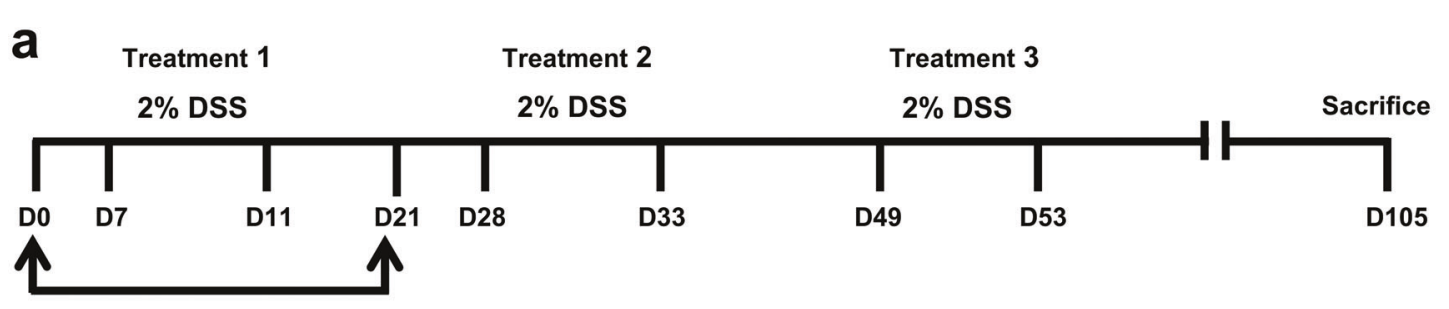

$12.5 \mathrm{mg} / \mathrm{Kg}$ AOM I.P.

b
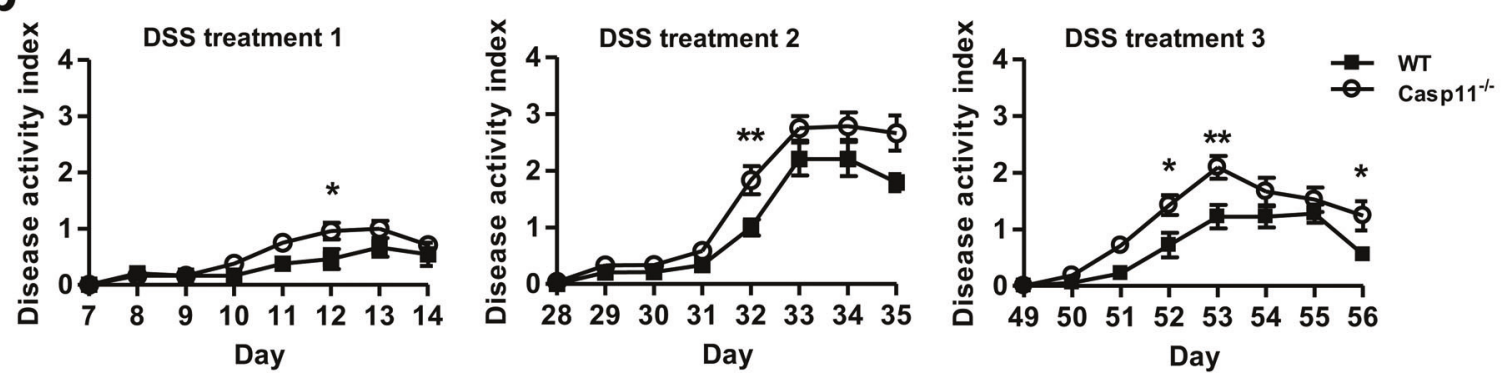

C
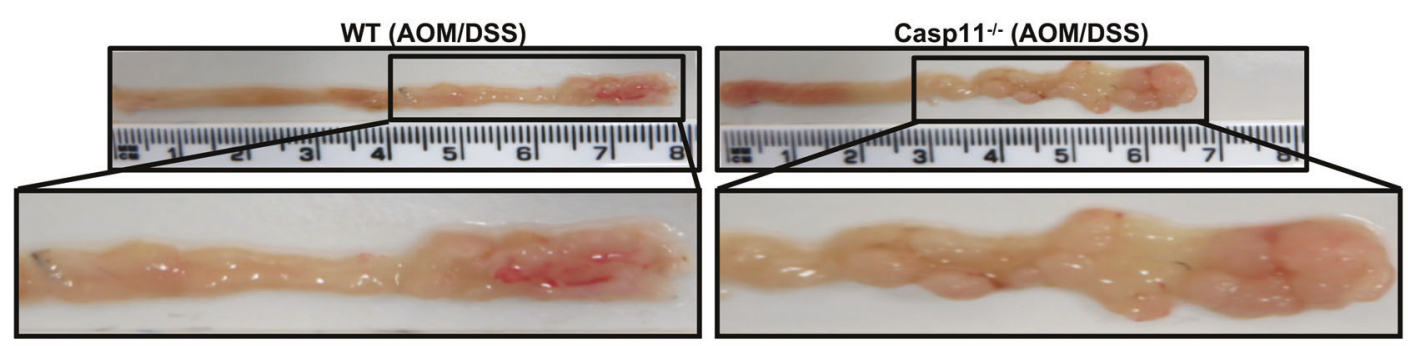

d
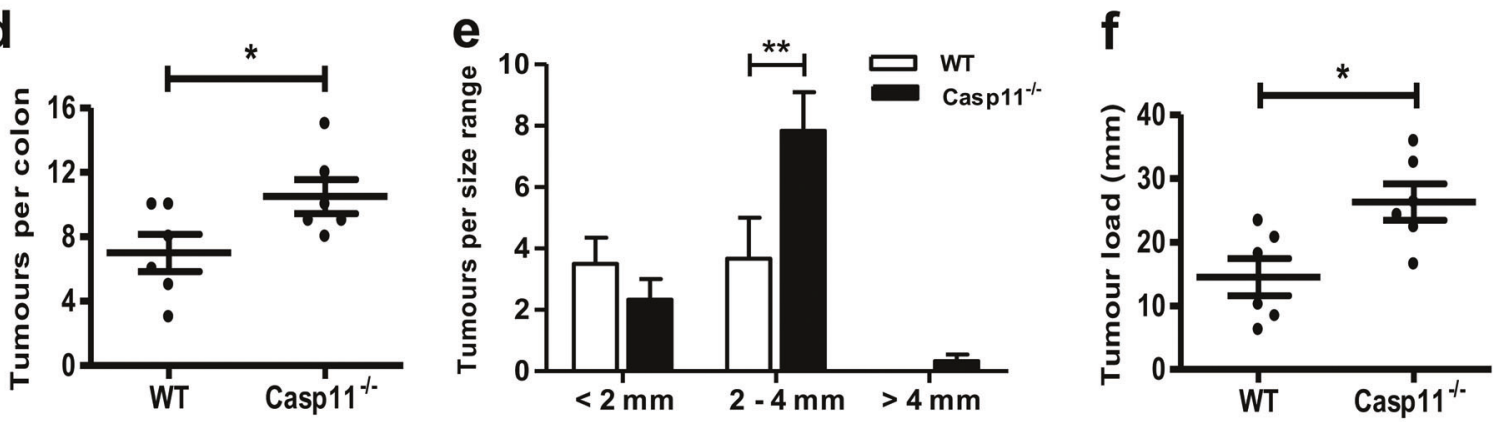

g

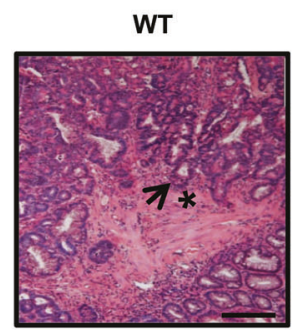

Intramucosal carcinoma

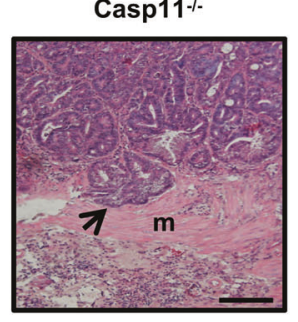

Invasive

h

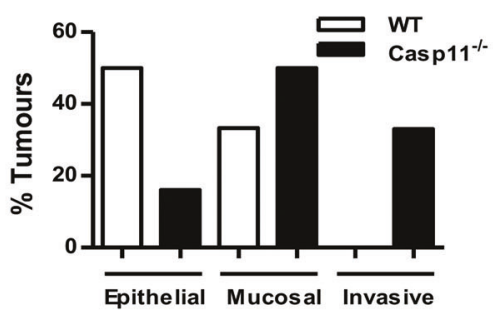

protective role for caspase-11 and the non-canonical inflammasome in controlling mucosal integrity during DSS-induced colitis [12, 16, 17]. The DSS-colitis phenotype of $\mathrm{Caspl1}^{-/-}$mice is very similar to those of mice deficient in canonical inflammasome components, caspase1, ASC and NLRP3 [4, 18]. In humans, intestinal expression of caspases- 4 and -5 , orthologs of murine caspase-11, is significantly enhanced in colon tissue from IBD and CRC 
Fig. 1 Increased susceptibility of Casp11-/- mice to experimental CAC. a Schematic representation of the AOM/DSS administration regime to induce tumour progression. WT and $\operatorname{Casp}_{11^{-1-}}$ mice were injected intraperitoneally (IP) with $12.5 \mathrm{mg} / \mathrm{kg}$ AOM on days 0 and 21 followed by 3 cycles of 2\% DSS (w/v). Mice were culled on day 105 . b Disease activity index (combination of weight loss, stool consistency and intestinal bleeding scores) during each DSS administration. Data represent mean \pm SEM of $n=6$ AOM/DSS-treated mice for both groups; $* p<0.05$; ** $p<0.01$ (two-way ANOVA followed by the Bonferroni post test). c Representative macroscopic view of the AOM/ DSS-derived tumours in AOM/DSS-treated WT and Casp11 $1^{-1-}$ mice, enhanced image magnification facilitates the visualisation of defined adenomas. d-f Total tumour numbers were macroscopically enumerated in AOM/DSS-treated WT and Casp $11^{-1-}$ whole colons (d); measured and assigned a size range (e); and each mouse was subsequently assigned a tumour load (calculated by the addition of each tumour diameter) (f). Data represent mean \pm SEM of $n=6$ AOM/DSStreated mice for both groups; $* p<0.05$; $* * p<0.01$ (two-tailed independent Student $t$-test). g Representative images of WT and Casp11 ${ }^{-1-}$ AOM/DSS-treated H\&E stained colon tissue displaying intramucosal carcinoma and invasive carcinoma (scale bar $=200 \mu \mathrm{m}$ ). $\mathbf{h}$ Tumours from AOM/DSS-treated mice displaying epithelial, mucosal and invasive carcinomas, as a percentage of total tumours. H\&E staining; *muscularis mucosae; m, submucosa

patients [19]. Of note, epithelial expression of caspases-4 and -5 is exclusive to neoplastic colon tissue, and have potential as CRC biomarkers [19]. Thus, it is tempting to speculate that inflammatory caspases- 4 and -5 have a functional role during CRC.

Canonical inflammasome components, including caspase-1, ASC, NLRP3, NLRC4, NLRP1 and AIM2 have been shown to significantly attenuate inflammation and tumorigenesis in the murine CAC model [4, 18, 20-27]. With the exception of AIM2, the protective effects of inflammasomes during CAC occurs by regulating IL-18 production [4, 18, 20-25]. IL-18 secretion stimulates intestinal epithelial cell (IEC) regeneration and repair, and improves colon barrier function, which may explain the enhanced susceptibility of inflammasome-deficient mice to CAC [28]. Given the essential role for caspase-11 and the non-canonical inflammasome in the regulation of IL-18 production during DSS-colitis, we hypothesised that caspase-11 may also be essential for the production of intestinal IL-18 and IL- $1 \beta$ during CAC. To explore this hypothesis, we investigated the role of caspase-11 in the pathogenesis of CAC using the AOM-DSS model in Caspl1 $1^{-1-}$ mice.

This study demonstrates that caspase-11-deficient mice have an increased susceptibility to colitis-associated tumourigenesis. We show reduced intestinal IL- $1 \beta$ production and impaired STAT1 activation in AOM-DSS-treated Casp $11^{-1-}$ mice, consistent with decreased IEC death and increased IEC proliferation, when compared with similarly treated WT mice. We also demonstrate a requirement for caspase-11 during IL-1 $\beta$-mediated STAT1 activation in IECs. Collectively, our data demonstrate that caspase-11, and a functional non-canonical inflammasome, is required for the tumour suppressive role of STAT1 during experimental CAC.

\section{Results}

\section{Increased susceptibility of Casp11 ${ }^{-I-}$ mice to experimental CAC}

To determine whether the increased intestinal inflammation in Casp $11^{-/-}$mice led to increased colitis-associated colorectal tumourigenesis, Casp11 $1^{-1-}$ mice and their wild-type (WT) littermates were treated with the DNA methylating agent AOM $(12.5 \mathrm{mg} / \mathrm{kg})$ and three cycles of $2 \%$ DSS interspaced with normal drinking water (Fig. 1a). Mice were monitored for clinical signs of gastrointestinal disease (weight loss, diarrhoea and rectal bleeding, Supplementary Figure 1a). Casp $11^{-1-}$ mice consistently scored higher than their WT counterparts for diarrhoea and rectal bleeding, which contributed to overall higher disease activity scores across each of the DSS treatments (Fig. 1b, Supplementary Figure 1b). At the experimental endpoint, 15 weeks after the initial AOM injection, macroscopic evaluation of dissected and washed colons indicated the development of welldefined tumours in WT and Casp11 $1^{-1-}$-treated mice (Fig. 1c). The majority of tumours were located in the distal colon in both genotypes, although $\operatorname{Casp11}^{-/-}$mice also displayed numerous tumours in the mid-colon region (Fig. 1c). Further evaluation of tumour number and size revealed that tumours in Casp11 $1^{-1-}$ mice were larger and of higher incidence than those observed in WT mice, with a significantly greater tumour load in $\mathrm{Caspl1}^{-1-}$ mice (Fig. 1df). Histopathological examination revealed that tumours

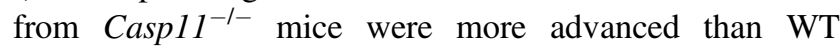

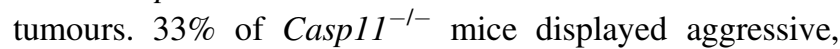
invasive carcinomas (as indicated by submucosal invasion), which was not observed in WT mice (Fig. 1g, h). Furthermore, tumour tissue from $50 \%$ of $\mathrm{Caspl1}^{-1-}$ mice displayed extension of severely dysplastic regions below the muscularis mucosae, compared with 33\% of WT mice (Fig. $1 \mathrm{~g}, \mathrm{~h}$ ). These observations demonstrate that caspase- 11 is protective during intestinal carcinogenesis.

\section{Increased angiogenesis and reduced cell death characterise the enhanced carcinogenesis phenotype of Casp11 ${ }^{-I-}$ mice}

Angiogenesis is a pre-requisite for tumour invasion and inhibition/depletion of caspase-1 has been implicated in the improvement of angiogenesis and blood supply in functional angiogenesis murine models $[29,30]$. As substantially higher levels of invasive tumours were observed 
a

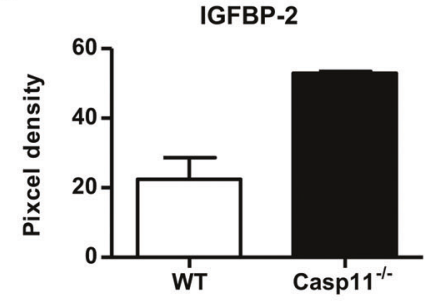

Osteopontin

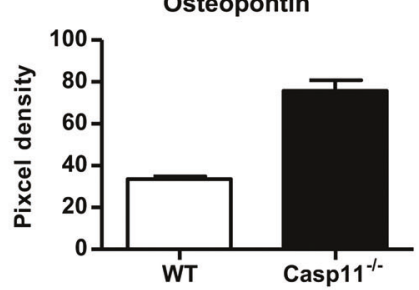

Platelet Factor 4

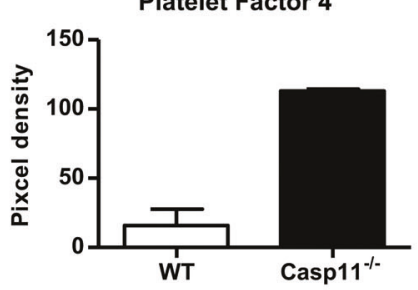

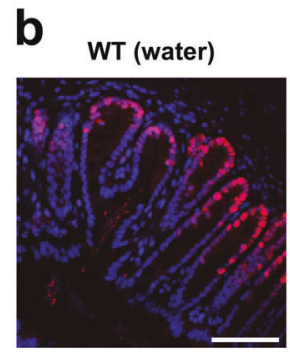
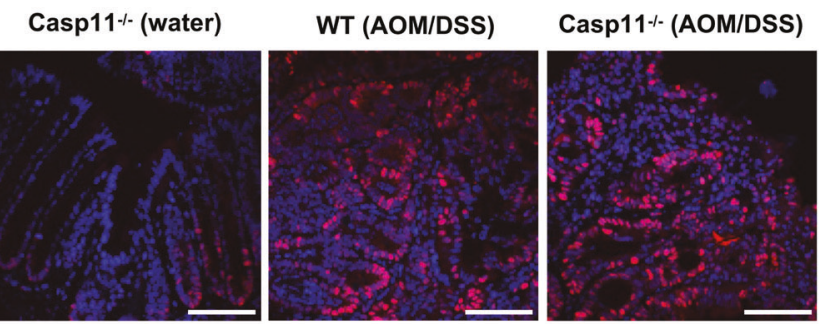

C
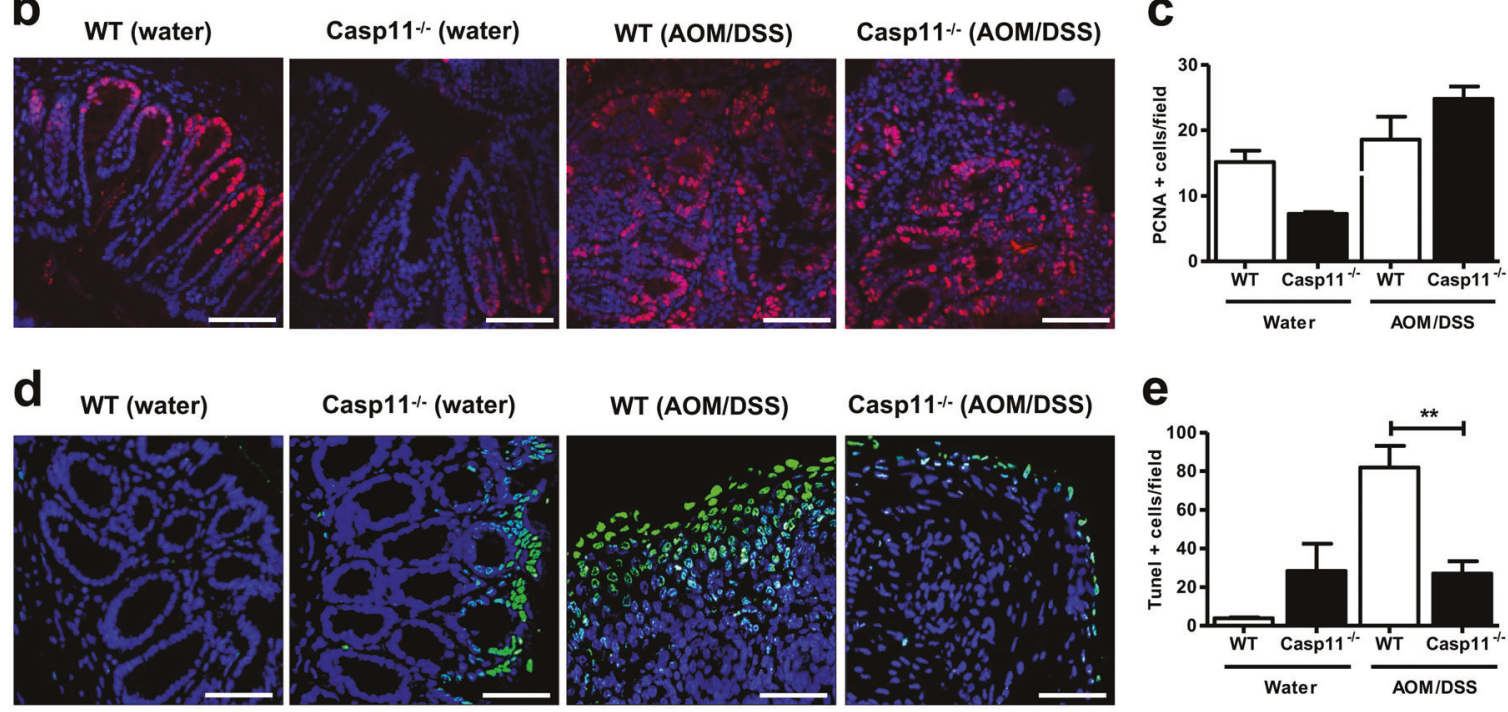

Casp11\% (water)
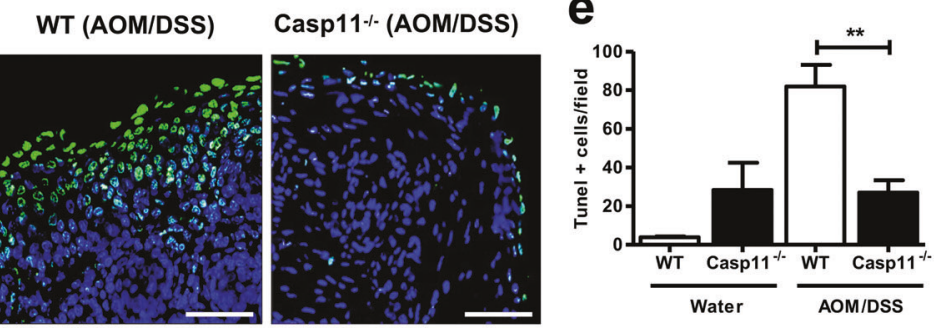

Fig. 2 Increased angiogenesis and reduced cell death characterise the enhanced CAC phenotype of Casp11 $1^{-/-}$mice. a Semi-quantitative IGFBP-2, osteopontin and platelet factor 4 expression in AOM/DSS-

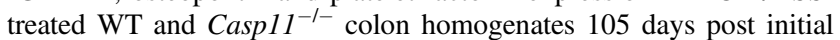
AOM IP injection, as determined using an angiogenesis proteome profiler array and ImageJ densitometry. Data represent mean \pm SEM of

in AOM-DSS-treated Casp $11^{-1-}$ colons, compared to WT (Fig. 1g, h), we looked for differences in expression of angiogenesis-related proteins in colons from the two treatment groups. An angiogenesis proteome profile array revealed enhanced expression of the proteins IGFBP-2, osteopontin (OPN) and platelet factor 4 (PF4) in Casp11 $1^{-1-}$ homogenates (Fig. 2a, Supplementary Figure 2). OPN expression has been previously shown to enhance colon cancer cell growth, invasion and angiogenesis [31], and both IGFBP-2 and PF4 have been identified as biomarkers for the early detection of colorectal cancer [32, 33]. These findings suggest that caspase-11 regulates the expression of proteins associated with early stage angiogenesis during $\mathrm{CRC}$, and strengthen the evidence for a protective role for caspase-11 during colorectal cancer.

The colonic mucosa of individuals with colon carcinoma is hyper-proliferative when compared with that from normal individuals [34]. To determine the consequence of caspase11 deficiency on intestinal epithelial cell (IEC) proliferation during established CAC, proliferating cell nuclear antigen (PCNA) staining was assessed in distal colon sections from $n=2$ AOM/DSS-treated mice for both groups. b-e Representative immunofluorescent images of proliferating cell nuclear antigen (PCNA) (b) and TUNEL (d) positive epithelial cells in distal colon tissue sections of AOM/DSS-treated WT and Casp11 $1^{-1-}$ mice (scale bars $=50 \mu \mathrm{m}(\mathbf{b})$ and $500 \mu \mathrm{m}(\mathbf{d}))$. c, e Quantification of PCNA (c) and TUNEL (e) positive epithelial cells shown in $\mathbf{b}$ and $\mathbf{d}$

CAC in WT and Casp $11^{-/-}$mice. Results show a moderate, although not significant, increase in PCNA-positive IECs in Casp $11^{-1-}$ colon sections (Fig. 2b, c). Consistent with our previous observations [12], less basal intestinal cell proliferation was observed in untreated Casp11 $1^{-/-}$colons, compared to WT controls (Fig. 2b, c). We also find that overall proliferation rates in healthy WT murine colons are similar to those of AOM-DSS-treated WT colons (Fig. 2c), which has been observed by other groups [4, 27, 35]. This may be explained by the fact that rapid proliferation of Lgr5 ${ }^{+}$stem cell-generated transit amplifying (TA) cells occurs in the lower half of crypts in healthy WT colons (Fig. 2b) [36]. However, DSS causes mucosal damage, destroying Lgr5 $^{+}$ cells and altering normal proliferation rates [37], while transformed IECs emerge as the rapidly proliferating population.

Given the key role of caspase-11 in driving pyroptotic and other cell death pathways [8, 9, 38, 39], we addressed whether the increased tumourigenic load observed in AOMDSS-treated Casp $11^{-1-}$ mice could be explained by reduced levels of IEC death. Both pyroptotic and apoptotic modes of 
a

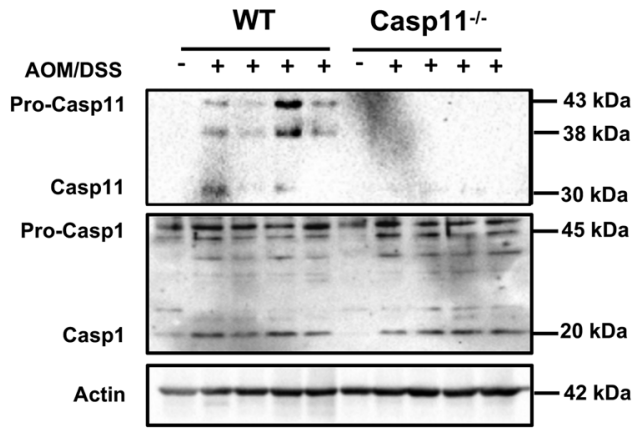

C

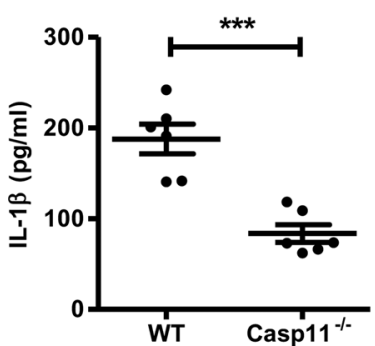

g

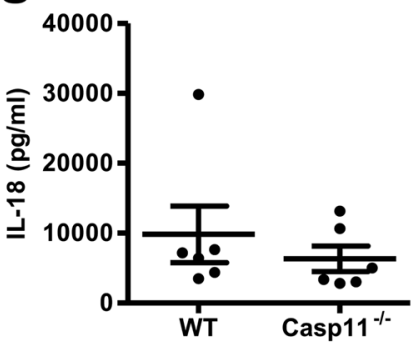

k

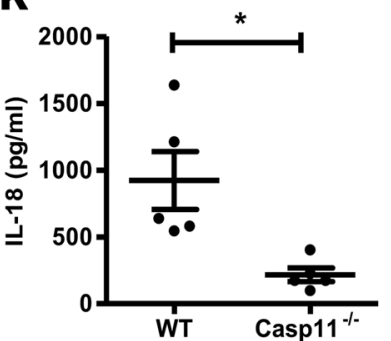

d

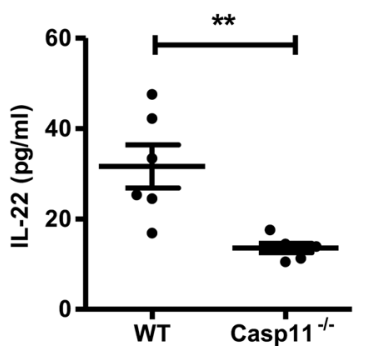

h

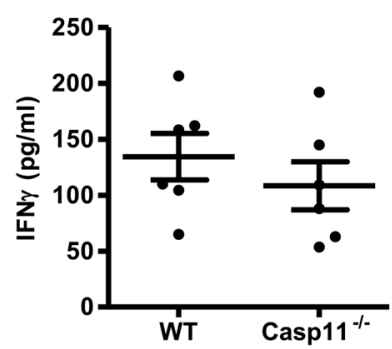

I

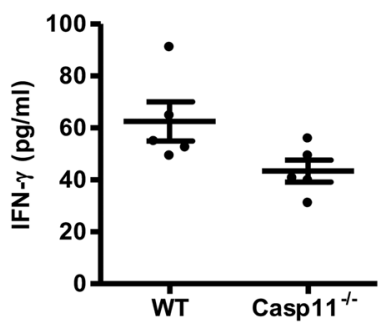

Fig. 3 Reduced IL-1 $\beta$ production in Casp $11^{-1-}$ mice during advanced stages of CAC. a Western blot analysis of caspase-11 and caspase-1 expression in colon homogenates from AOM/DSS-treated WT and Casp $11^{-1-}$ mice on day 105 post initial AOM IP injection. Each lane represents an individual mouse. b Analysis of relative Casp11 mRNA expression in AOM/DSS-treated C57BL/6 J colon tissue using the published dataset GSE64658. Data represent mean \pm SEM; $* p<0.05$;

b

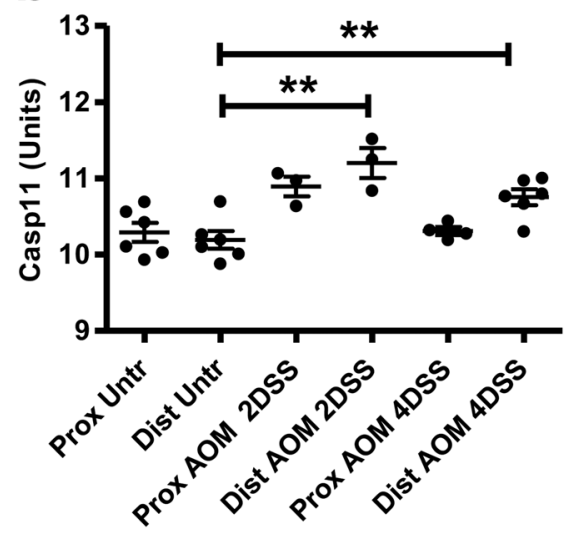

e

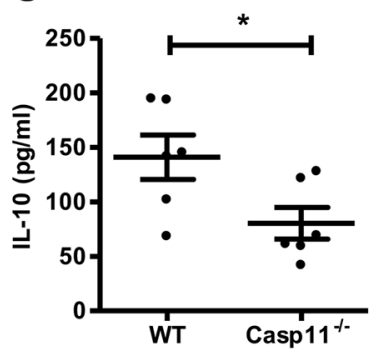

f

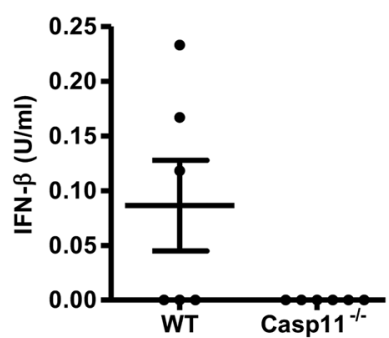

i
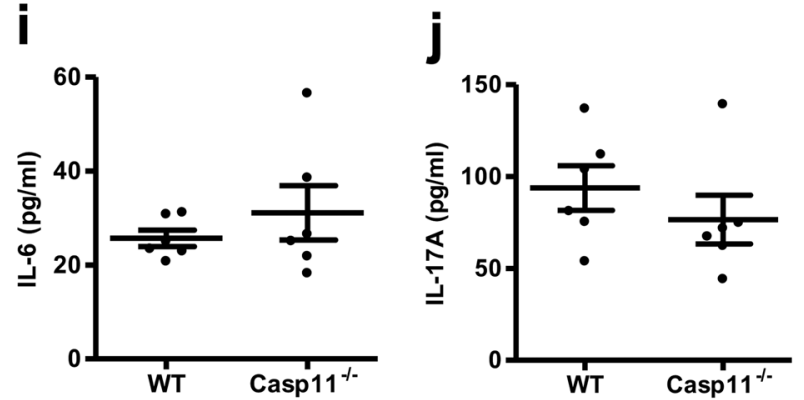

$* * p<0.01$; (two-tailed independent Student $t$-test). c-l Cytokine levels in colon homogenates of AOM/DSS-treated WT and Casp11 ${ }^{-1-}$ mice on day $105(\mathbf{c}-\mathbf{j})$ and day $21(\mathbf{k}, \mathbf{l})$ post initial AOM IP injection, as measured by ELISA. Data represent mean \pm SEM of $n=6(\mathbf{c}-\mathbf{j})$ and $n$ $=5(\mathbf{k}, \mathbf{l}) \mathrm{AOM} / \mathrm{DSS}$-treated mice for both groups; $* p<0.05$; ** $p<$ $0.01 ; * * *<0.001$ (two-tailed independent Student $t$-test) 
a

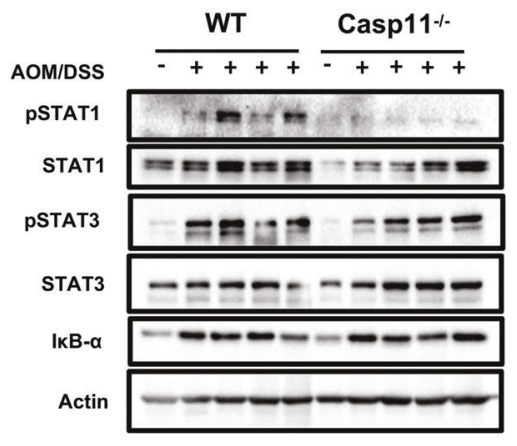

b

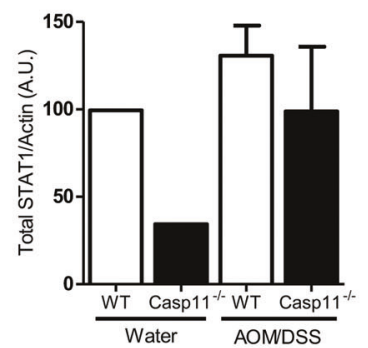

C

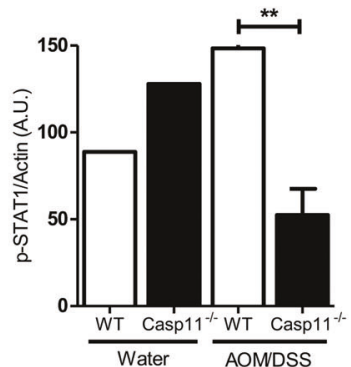

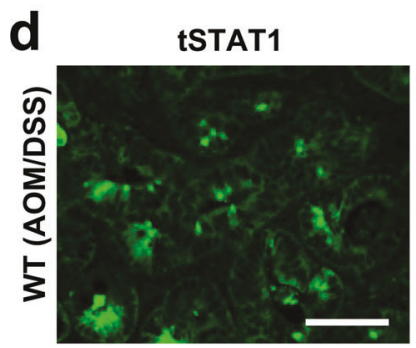
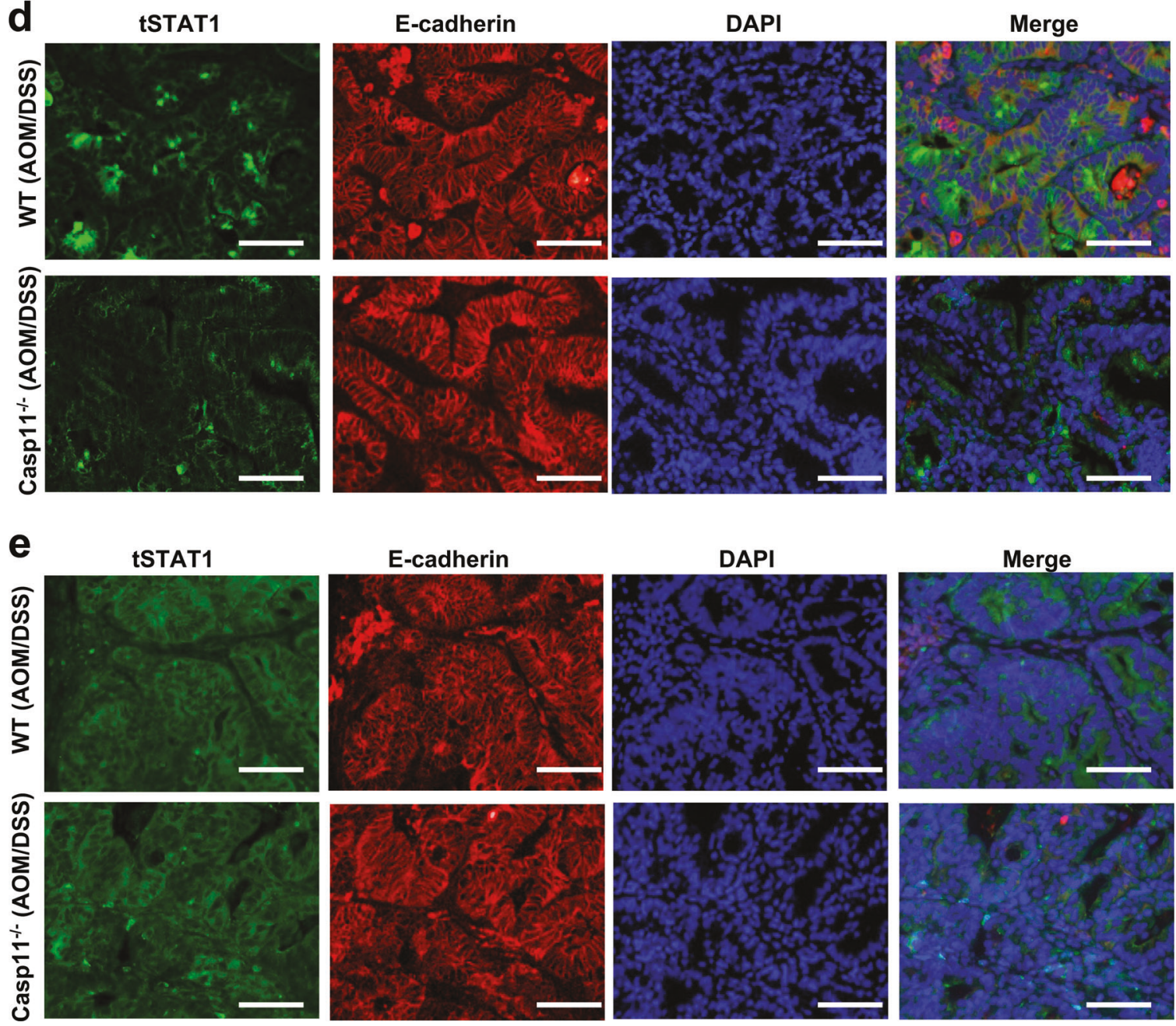

Fig. 4 Caspase-11 mediates STAT1 activation during CAC. a Western blot analysis of phosho-/total STAT1, STAT3, IкB $\alpha$, and actin (loading control) in colon homogenates from AOM/DSS-treated WT and Casp $11^{-/-}$mice on day 105 post initial AOM IP injection. Each lane represents an individual mouse. b Total STAT1 and $\mathbf{c}$ pSTAT1 expression densitometry (relative to actin) in colon homogenates from

cell death are detectable by TUNEL staining [40]. Distal colon sections of AOM-DSS-treated mice revealed a significantly lower number of TUNEL-positive IECs in
AOM/DSS-treated WT and Casp11 $1^{-1-}$ mice. $* * p<0.01$ (two-tailed unpaired t-test). Representative immunofluorescent images of d pSTAT1; and e total STAT1; co-stained for the epithelial marker Ecadherin (red) and DAPI (blue) in distal colon sections from AOM/ DSS-treated WT and Casp11 ${ }^{-1-}$ mice at day $105($ scale bar $=20 \mu \mathrm{m})$

Casp11 $1^{-1-}$ compared to WT colons, confirming the importance of caspase-11 in promoting cell death during advanced stages of CAC (Fig. 2d, e). 


\section{Reduced IL-1 $\beta$ production in Casp $11^{-1-}$ mice during advanced stages of CAC}

Colon homogenates from AOM-DSS-treated WT mice demonstrate elevated caspase-11 expression at the 15 week experimental endpoint (Fig. 3a). This is in agreement with the enhanced expression of human caspase 4 and 5 during CRC [19], strongly suggesting an active involvement of inflammatory caspases during CAC. As canonical inflammasome activity is not affected by caspase- 11 deficiency [12], we expect that a certain level of caspase-1 activation will still occur in AOM-DSS-treated Casp11 $1^{-1-}$ colons. Fig. 3a shows that similar levels of full length and processed (p20) caspase-1 subunits are apparent in both WT and Casp $11^{-1-}$ colon homogenates. However, conclusions regarding caspase- 1 activity cannot be made from this result, as a recent report suggests that caspase- 1 immunoblots are not representative of its activity, and that processed caspase1 subunits (p20/p10 - previously considered to be active), in fact represent inactivated caspase-1 subunits [41]. Consistent with our findings, mRNA analysis from an independent AOM-DSS trial [42] also demonstrates significantly elevated caspase-11 mRNA levels in distal colons following both two and four rounds of DSS treatment (Fig. 3b).

Cytokine expression was quantified by ELISA in WT vs. Casp $11^{-/-}$mice during CAC, using colon homogenates from 15 week endpoints. Data demonstrate that colons from Casp $11^{-/-}$mice have significantly less IL-1 $\beta$, IL-22 and IL10 than their WT littermate controls (Fig. 3c-e). As the tumour load was higher in Casp11 $1^{-1-}$ colons, compared to WT, it is possible that enhanced tumorigenesis may contribute to the cytokine differences observed between genotypes. However, defective IL- $1 \beta$ production during CAC in Casp $11^{-1-}$ mice is consistent with previous studies evaluating inflammasome signalling during $\mathrm{CAC}$, including Asc ${ }^{-1-}$, Caspl $^{-1-} / 11^{-1-}$ and Nlrp1 ${ }^{-1-}$ mice $[20,25]$. The specificity of these data are demonstrated by the observation that no significant differences in colon levels of IFN $\beta$ IL-18, IL- $1 \alpha$ IFN $\gamma$, IL- 6 or IL-17a were detected at this advanced stage of CAC (Fig. 3f-j). Importantly, decreased IL-18, and to a lesser extent IFN $\gamma$, production was evident in Casp11 ${ }^{-1-}$ mice at 2 weeks (Fig. 3k, 1), which may be ultimately responsible for defective IL-22 production observed in these mice during both experimental colitis and CAC [43]. Thus, during advanced stages of CAC, intestinal IL- $1 \beta$ and IL-22 appear to be the cytokines most significantly affected by caspase-11 deficiency.

\section{Caspase-11 is required for STAT1 activation during CAC}

We have previously shown that STAT1 expression and phosphorylation levels are upregulated during DSS-colitis
[12]. STAT1 activation is also a consequence of CAC, and has been proposed as a mechanism for the protective effects of the NLRP3 inflammasome during CAC [4]. We therefore investigated the expression and activation status of STAT1 in AOM-DSS-treated WT and Casp11 $1^{-/}$colon homogenates, and observed a marked impairment of STAT1 activation in Casp11 $1^{-1-}$ colons of CAC-treated mice (Fig. $4 \mathrm{a}-\mathrm{c})$. In contrast, caspase-11-specific alterations were not observed in total or phosphorylated STAT3, or IкB expression levels (Fig. 4a). IF staining of WT colon tumour tissue indicated STAT1 activation in IECs during AOMDSS treatment, and confirmed the STAT1 activation deficiency in Casp $11^{-/-}$colon tumours (Fig. 4d). Total STAT1 levels appear to be similar in tumour tissue from WT and Casp $11^{-l-}$ colons (Fig. 4e). STAT1 activation appears to be occurring predominantly in tumorigenic epithelial cells, as F4/80-positive immune cells did not express phosho-STAT1 (Supplementary Figure 3). These results suggest that the protective role of caspase- 11 during experimental CAC is mediated by anti-tumorigenic STAT1 signalling in IECs.

\section{Impaired STAT1 activation and increased IEC proliferation associates with caspase-11 deficiency during development of CAC}

To further explore the association between caspase-11 and STAT1 signalling during CAC, we examined whether deficiencies in STAT1 activation also occurred at earlier stages of CAC. To address this experimentally, we terminated the AOM-DSS protocol (Fig. 1a) at 6 weeks, a stage at which adenomas were developing, but were not yet macroscopically detectable. Western blot analysis of colon homogenates showed increased expression of caspase- 11 at this stage of CAC development (Fig. 5a). As observed in the 15 week AOM-DSS trial, Casp $11^{-1-}$ mice displayed higher disease activity scores than their WT littermates during DSS treatments (Fig. 5b, Supplementary Figure 4). Symptom variability can occur between DSS treatments [37], and we observed a high overall disease activity in DSS treatment 1 of this trial (Fig. 5b), due to increased detection of blood in the faecal pellets of Casp $11^{-1-}$ mice (Supplementary Figure 4c).

Analysis of WT and Casp11 $1^{-1-}$ colons at the 6 week tumour development stage revealed significantly increased IEC proliferation in Casp11 $1^{-/-}$colons compared with treatment matched WT colons, as assessed by staining and histological evaluation of the proliferation markers PCNA (Fig. 5c, d) and Ki67 (Fig. 5e, f). In contrast to 15 week colons, TUNEL staining revealed no significant differences in the levels of IEC death between WT and Casp11 ${ }^{-1-}$ colons at 6 weeks (Supplementary Figure 5a, b). Alterations in angiogenic markers were not observed at the tumour development stage (Supplementary Figure 5c, d). 
a

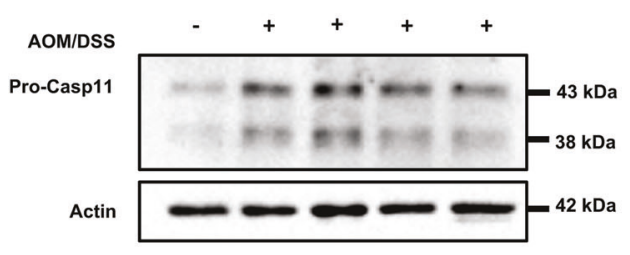

C
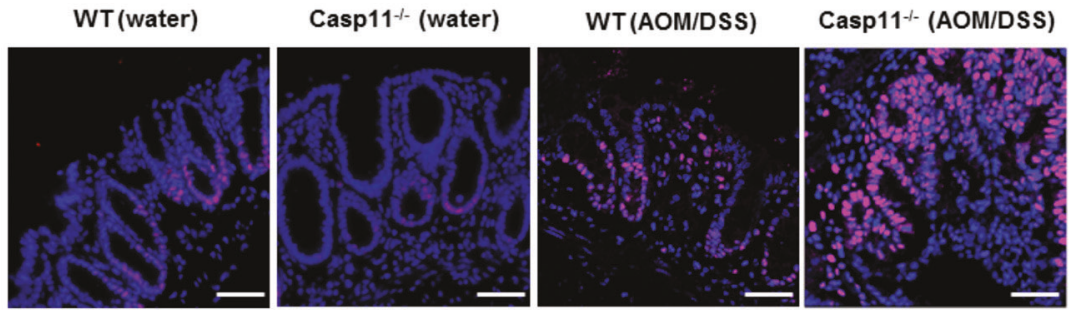

b DSs treatment 1

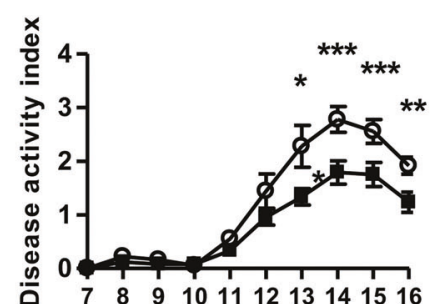

Day

e
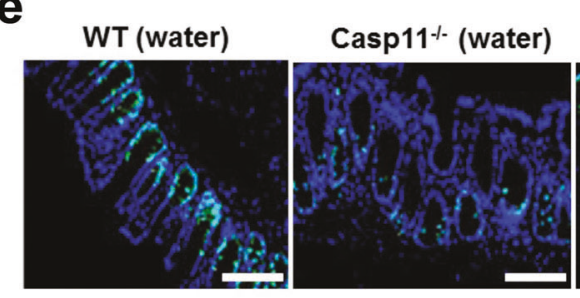

g
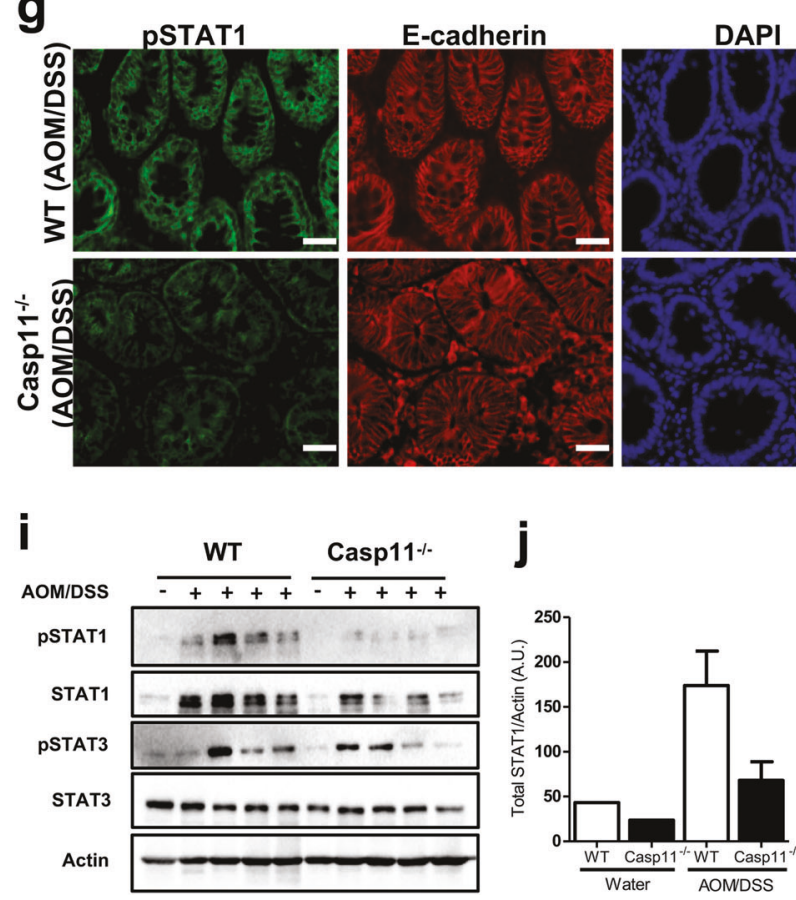

Total and phospho-STAT1 expression levels were also assessed, to determine whether the anti-proliferative role for caspase-11 observed in IECs during CAC development was
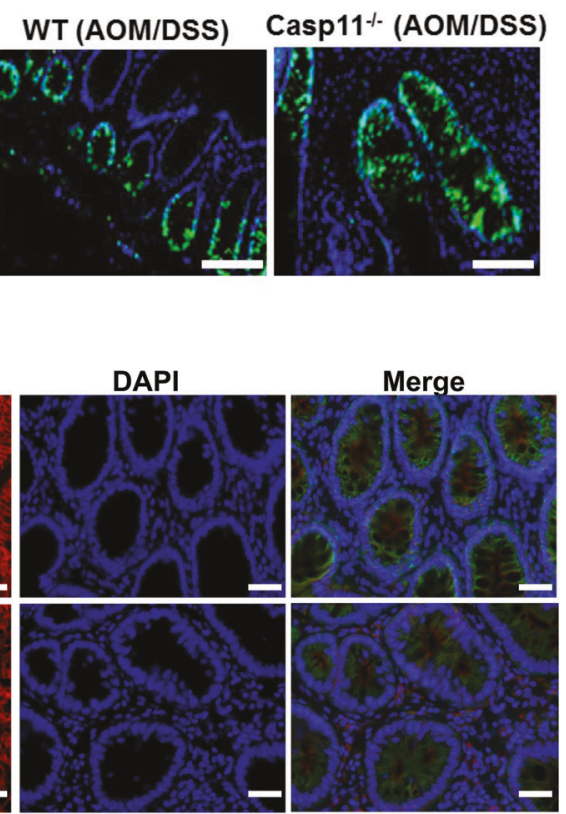

k

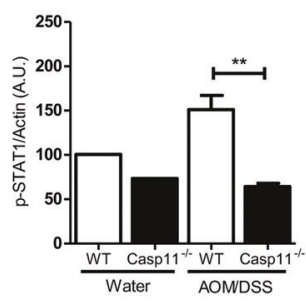

$\mathbf{f}$

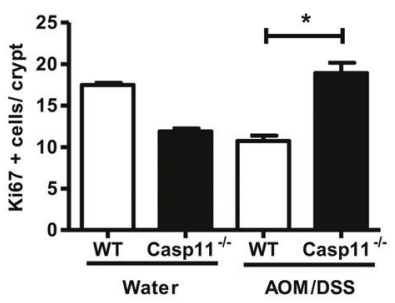

h

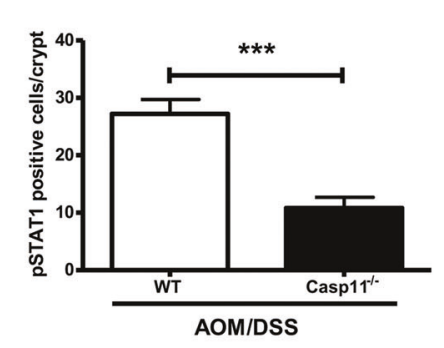

DSS treatment 2

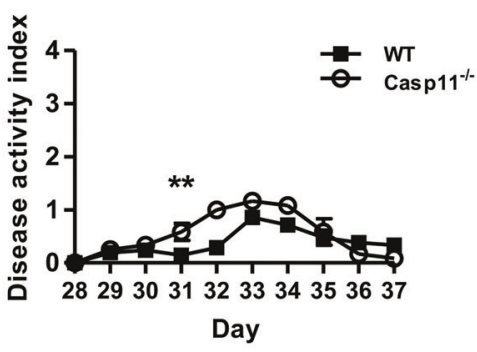

d

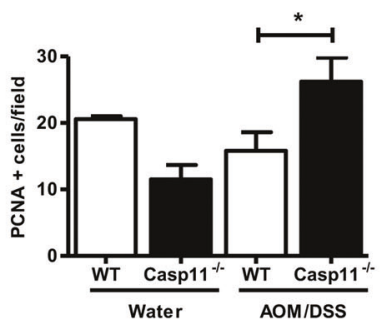

consistent with the anti-proliferative function of STAT1. IF staining in dysplastic colon tissue showed that less STAT1 activation occurred during tumour development in Casp11 
Fig. 5 Impaired STAT1 activation and increased IEC proliferation are evident in Casp $11^{-/-}$mice during CAC development. a Western blot revealing caspase-11 expression in colon homogenates of C57BL/6 mice on day 42 post initial AOM IP injection. Each lane represents an individual mouse. b Disease activity index during each DSS administration. Data represent mean \pm SEM of $n=6$ (WT) and $n=5$ $\left(\right.$ Casp 11 1 $\left.{ }^{-l-}\right)$ AOM/DSS-treated mice; $* p<0.05 ; * * p<0.01 ; * * * p<$ 0.001 (two-way ANOVA followed by the Bonferroni post test). c-f Representative immunofluorescent images of proliferating cell nuclear antigen (PCNA) (c); and Ki67 (e); positive epithelial cells in distal colon tissue sections of AOM/DSS-treated WT and Caspl1 ${ }^{-1-}$ mice (scale bars $=50 \mu \mathrm{m}, \mathbf{c}$ and $100 \mu \mathrm{m} \mathbf{e}$ ). Quantification of PCNA (d) and Ki67 (f) positive epithelial cells shown in $\mathbf{d}$ and $\mathbf{f}$. Data represent mean \pm SEM of $n=6(\mathrm{WT})$ and $n=5\left(\right.$ Casp 11 $\left.^{-1-}\right)$ AOM/DSS-treated mice; ${ }^{*} p<0.05$ (two-tailed independent Student $t$-test). g Representative IF images of distal colon sections from AOM/DSS-treated WT and $\operatorname{Casp}_{11^{-1-}}$ mice at day 42, stained for pSTAT1, epithelial marker E-cadherin and DAPI (Scale bar $=20 \mu \mathrm{m}$ ). $\mathbf{h}$ Quantification of pSTAT1 positive IECs cells in IF stained colon sections. (i) Western blot analysis of STAT1 and STAT3 activation in colon homogenates from AOM/DSS-treated WT and Casp $11^{-1-}$ mice on day 42 post initial AOM IP injection. Each lane represents an individual mouse. Expression dfensitometry (relative to actin) of $\mathbf{j}$ total STAT1 and $\mathbf{k}$ pSTAT1; in colon homogenates from AOM/DSS-treated mice on day 42 post initial AOM IP injection. ${ }^{* *} p<0.01$ (two-tailed unpaired $t$ test)

-l- mice, compared to WT (Fig. 5 g, h; Supplementary Figure 6). Immunoblot results confirm this observation, revealing that STAT1 activation is also caspase-11 dependent at the tumour development stage (Fig. 5i-k). Total STAT1 expression analysis shows that there is also a trend towards lower total STAT1 levels in Casp11 ${ }^{-1-}$ colons (Fig. 5i, j). This observation is not surprising, as STAT1 activation has been shown to positively regulate its own expression [44]. These data support the hypothesis that caspase-11 mediates anti-tumourigenic STAT1 activity during experimental CAC.

\section{STAT1 activation in primary murine macrophages is regulated by caspase- 11}

The findings presented in this study identify IL-1 $\beta$ and STAT1 as the key cytokine and transcription factor, respectively, affected by caspase-11 deficiency during established CAC. The defective IL- $1 \beta$ production observed suggest a role for a functional non-canonical inflammasome during CAC. However, a link between non-canonical inflammasome activation and STAT1 signalling pathways is not well characterised. IFN $\gamma$ represents an obvious link between STAT1 and caspase-11, as it drives STAT1dependent caspase-11 expression [45]. No significant differences in IFN $\gamma$ levels were detected in Casp $11^{-1-}$ colon homogenates at the 6 or 15 week stages of CAC development (Fig. 3h, 1), however Casp $11^{-1-}$ colon explants taken 3 weeks into the AOM-DSS protocol secreted significantly less IFN $\gamma$ than their WT counterparts (Fig. 6c). Although impaired STAT1 activation appears to occur predominantly in Casp $11^{-1-}$ IECs during colorectal tumorigenesis, we sought to determine whether caspase-11 also has the ability to regulate STAT1 activity in primary macrophages. Primary bone marrow derived macrophages (BMDM) from WT and Casp $11^{-1-}$ mice were stimulated with IFN $\gamma$, LPS, or IL-1 $\beta$ and monitored for Phospho-STAT1 over $24 \mathrm{~h}$ (Fig. 6d-f). As expected, STAT1 phosphorylation was induced within $30 \mathrm{~min}$ of IFN $\gamma$ stimulation in both WT and Casp 11 -I- BMDM, and caspase-11 was upregulated within $4 \mathrm{~h}$ in WT BMDM only (Fig. 6d). Phospho-STAT1 levels decreased after $2 \mathrm{~h}$ but a moderate, sustained expression remained up to $24 \mathrm{~h}$. This STAT1 activity was reduced in Casp 11 $1^{-/-}$BMDM, suggesting that sustained IFN $\gamma$-driven STAT1 activity is partially dependent on caspase-11 (Fig. 6d). LPS stimulation resulted in significant STAT1 activation within $2 \mathrm{~h}$, which decreased by $8 \mathrm{~h}$. Similar to the effect of IFN $\gamma$, LPS-induced STAT1 activation was impaired in Casp $11^{-/-}$BMDM, supporting a role for caspase-11 in the regulation of this STAT1 activation pathway (Fig. 6e). Data shown in Fig. 6f demonstrate that IL-1 $\beta$ stimulated BMDM significantly upregulate caspase-11 within $2 \mathrm{~h}$, which is sustained until $24 \mathrm{~h}$. Phospho-STAT1 was detected within $4 \mathrm{~h}$ IL-1 $\beta$ stimulation in both WT and Casp $11^{-1-}$ BMDM, although no significant caspase-11 dependency for STAT1 activity was observed (Fig. 6f). Stimulations were also performed in BMDM from WT, Ifnar- and Ifn $\gamma$-deficient mice, to determine whether LPS and IL-1 $\beta$-stimulated activation of STAT1 were being mediated via Type I or II IFN signalling (Fig. 6g-i). Fig. $6 \mathrm{~g}$ confirms that IFN $\gamma$-stimulation results in STAT1 activation in both Ifnar $^{-1-}$ and If $n \gamma^{-1-}$ BMDM. Consistent with previous reports [12, 15], LPS-mediated induction of caspase-11 was significantly attenuated in Ifnar $^{-1-}$ BMDM (Fig. 6h). Similarly, STAT1 activation was unaffected in $I f n \gamma^{-1-}$ BMDM, but completely inhibited in Ifnar ${ }^{-/-}$BMDM, confirming that LPS-mediated STAT1 activation occurs via Type I IFNs (Fig. 6h). Analogous to LPS stimulations, IL-1 $\beta$-mediated upregulation of caspase-11 and STAT1 activation require type I IFN signalling (Fig. 6i). These experiments demonstrate that both LPS and IL-1 $\beta$ stimulate caspase-11 expression and STAT1 activation in BMDM via type I IFNs, and reveal a role for caspase-11 in LPS-mediated STAT1 activation in BMDM.

\section{Caspase-11 regulates LPS- and IL-1 $\beta$-mediated STAT1 activation in IECs}

To provide further evidence of a role for caspase-11 during CAC, we investigated whether stimulation with IFN $\gamma$, LPS and IL-1 $\beta$ was capable of upregulating caspase-11 in an established murine CRC cell line, CT26. The data shown in Fig. 7a confirm that CT26 cells respond to all stimuli by upregulating caspase-11. Interestingly, the kinetics of 

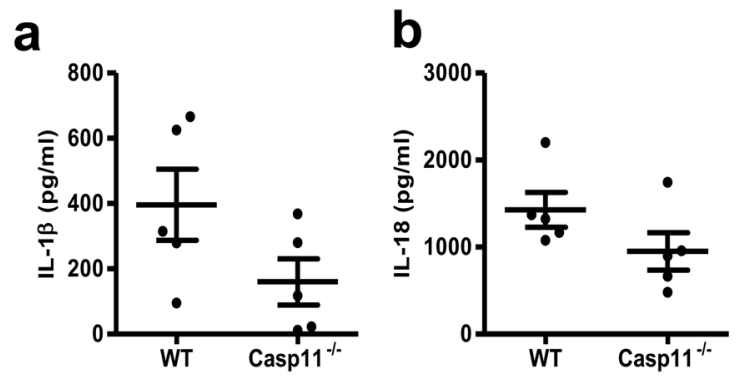

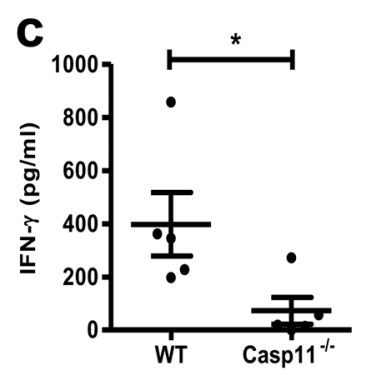

d

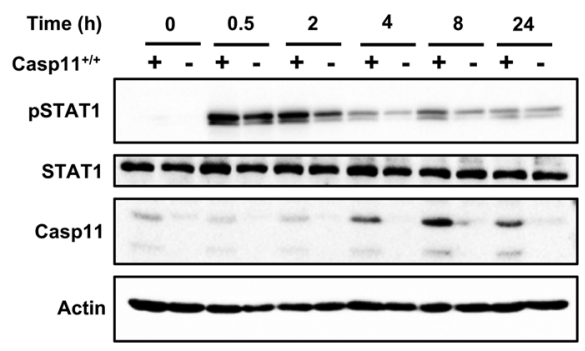

e

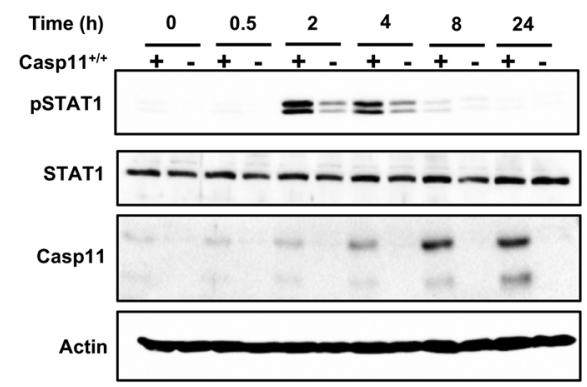

f

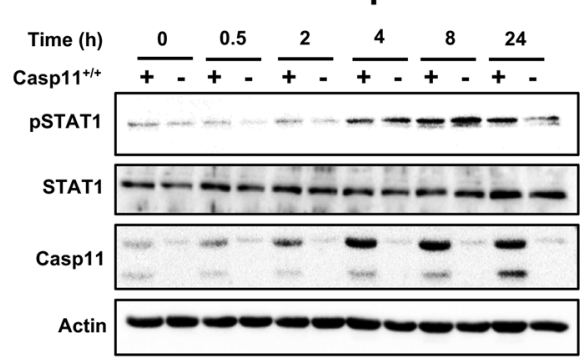

Fig. 6 Caspase-11 regulates STAT1 activation in primary murine macrophages. a-c Distal colon explant tissue isolated from AOM/ DSS-treated WT and Casp11 $1^{-1-}$ mice on day 21 post initial AOM IP injection were cultured for $20 \mathrm{~h}$. Supernatants were analysed for the production of IL-1 $\beta$ (a), IL-18 (b), and IFN- $\gamma(\mathbf{c})$, by ELISA; * $p<0.05$ (two-tailed independent Student $t$-test). d-f Western blot analysis of

caspase-11 upregulation following LPS and IL-1 $\beta$ stimulation closely correlate with those of STAT1 activation, supporting the notion that indirect STAT1 activation requires caspase-11 in colorectal carcinoma cells. g IFN-Y

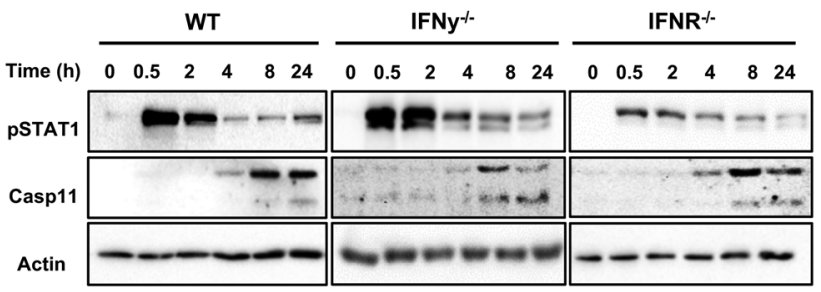

h LPS

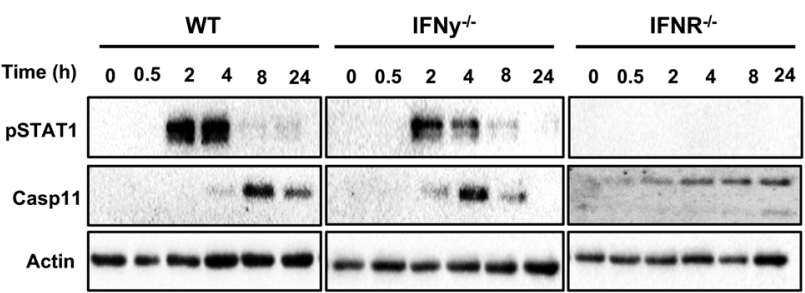

i IL-1 $\beta$

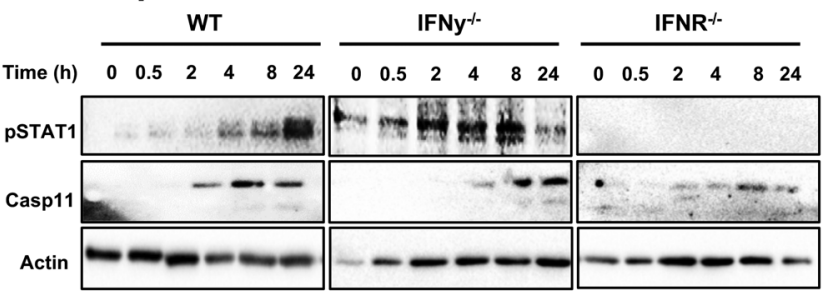

pSTAT1, total STAT1 and caspase-11 expression in WT and Casp11 BMDMs treated with IFN- $\gamma$ (d), LPS (e) and IL-1 $\beta$ (f). $\mathbf{g - i}$ Western blot analysis of STAT1 and caspase-11 expression in WT, Ifnar $^{-I-}$ and Ifn- $\gamma^{-I-}$ BMDMs treated with IFN- $\gamma(\mathbf{g})$, LPS (h) and IL$1 \beta$ (i). Blots are representative of more than three independent experiments

Having established a role for caspase-11 in STAT1 activity during CAC, and a link between IL-1 $\beta$ and STAT1 signalling in BMDM, we examined whether caspase-11 could also influence LPS or IL-1 $\beta$-mediated 
Fig. 7 Caspase-11 regulates LPS and IL- $1 \beta$-mediated STAT1 activation in IECs. a Western blot timecourse analysis of caspase-11, pSTAT1 and total STAT1 expression in cell lysates from CT26 colon cancer cells stimulated with IFN- $\gamma$, LPS and IL-1 $\beta$. b Representative western blot showing the relative expression levels of caspase-11, vimentin (mesenchymal cell marker) and keratin 18 (epithelial cell marker) in IECs and mesenchymal cell lysates isolated from WT colon tissue explants, which had been incubated in media or with LPS for $20 \mathrm{~h}$. c, e, $\mathbf{g}$ WT and Casp11 ${ }^{-I-}$ colon explant tissue were left untreated $(n=2)$ or treated $(n=$ 4) with IFN- $\gamma(\mathbf{c})$, LPS (e), and IL-1 $\beta$ (g). Following $20 \mathrm{~h}$ incubation, purified IEC lysates were analysed for expression of caspase-11, pSTAT1 and total STAT1 by western blot. d, f, h Densitometric analysis of pSTAT1 (relative to actin) in WT and Casp11 $1^{-1-}$ IEC lysates following stimulation with IFN$\gamma(\mathbf{d})$, LPS (f), and IL-1 $\beta$ (h). Data represent mean \pm SEM of $n=4$ (untreated) and $n=8$ (treated) WT and Casp11 $1^{-1-}$ mice; $* p<0.05 ; * * p<0.01$ (two-tailed independent Student $t$-test)

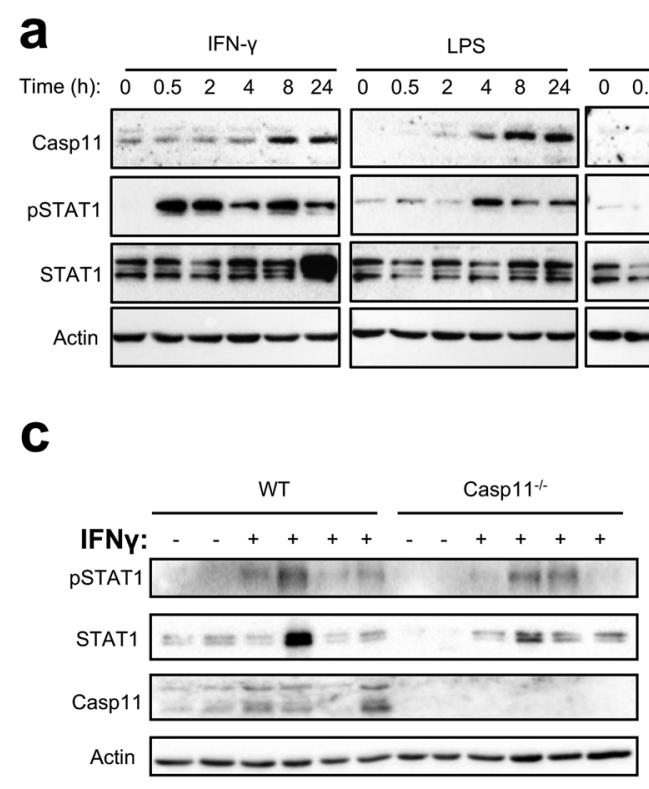

e

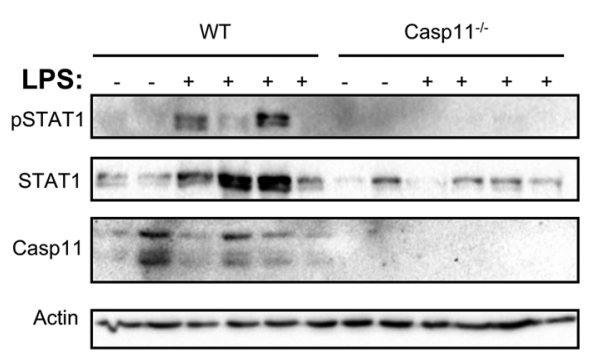

g

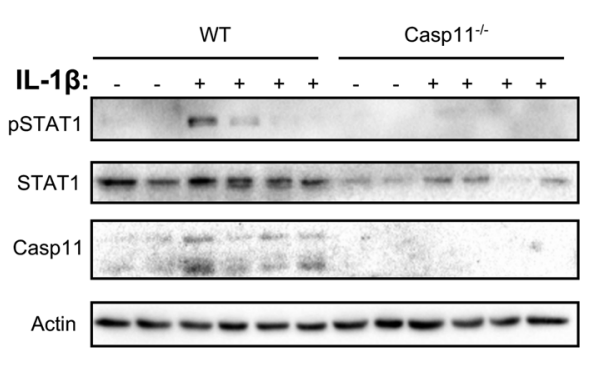

b

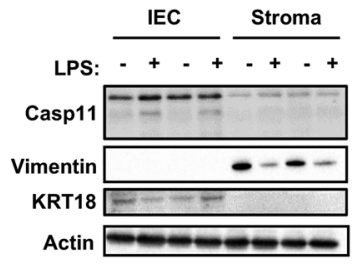

f LPS
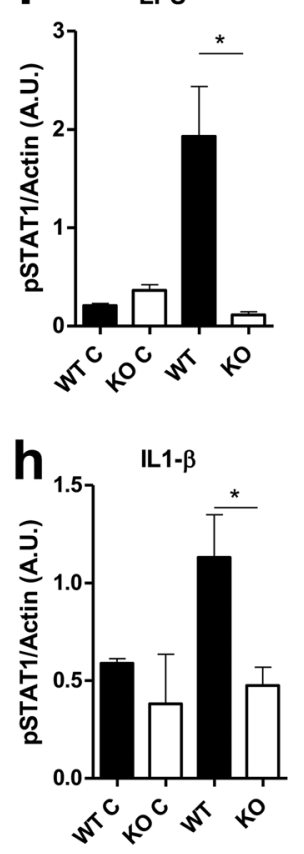

STAT1 activation in IECs. To establish a system whereby primary IECs can be stimulated in vitro, colon explant tissue $(1 \mathrm{~cm}$ sections) were incubated in media (alone or with stimulant) for $20 \mathrm{~h}$ before separating the IEC from the lamina propria (i.p.). The purity of IECs was confirmed by probing for cytokeratin-18, and the lamina propria cells by the presence of vimentin. Furthermore, LPS stimulation reveals that caspase-11 is selectively upregulated by IECs (Fig. 7b). To determine whether caspase-11 could regulate STAT1 activation pathways in IECs, colon explants from WT and Casp $11^{-1-}$ mice were stimulated with IFN $\gamma$, LPS and IL- $1 \beta$ before purifying IECs. Western blot revealed that STAT1 activation was similar in WT and Casp11 ${ }^{-1-}$ cells following IFN $\gamma$ stimulation (Fig. 7c, d), confirming that caspase-11 cannot influence direct STAT1 activation via IFN $\gamma$. In contrast, both LPS and IL-1 $\beta$-mediated activation of STAT1 was impaired in Casp11 $1^{-1-}$ IECs (Fig. 7e-h), suggesting that caspase-11 regulates crosstalk between LPS/ IL-1 $\beta$-mediated inflammation and STAT1 activation pathways in IECs.

Figure 8 provides a summary of the novel findings presented in this manuscript: a suppressive role for caspase- 11 


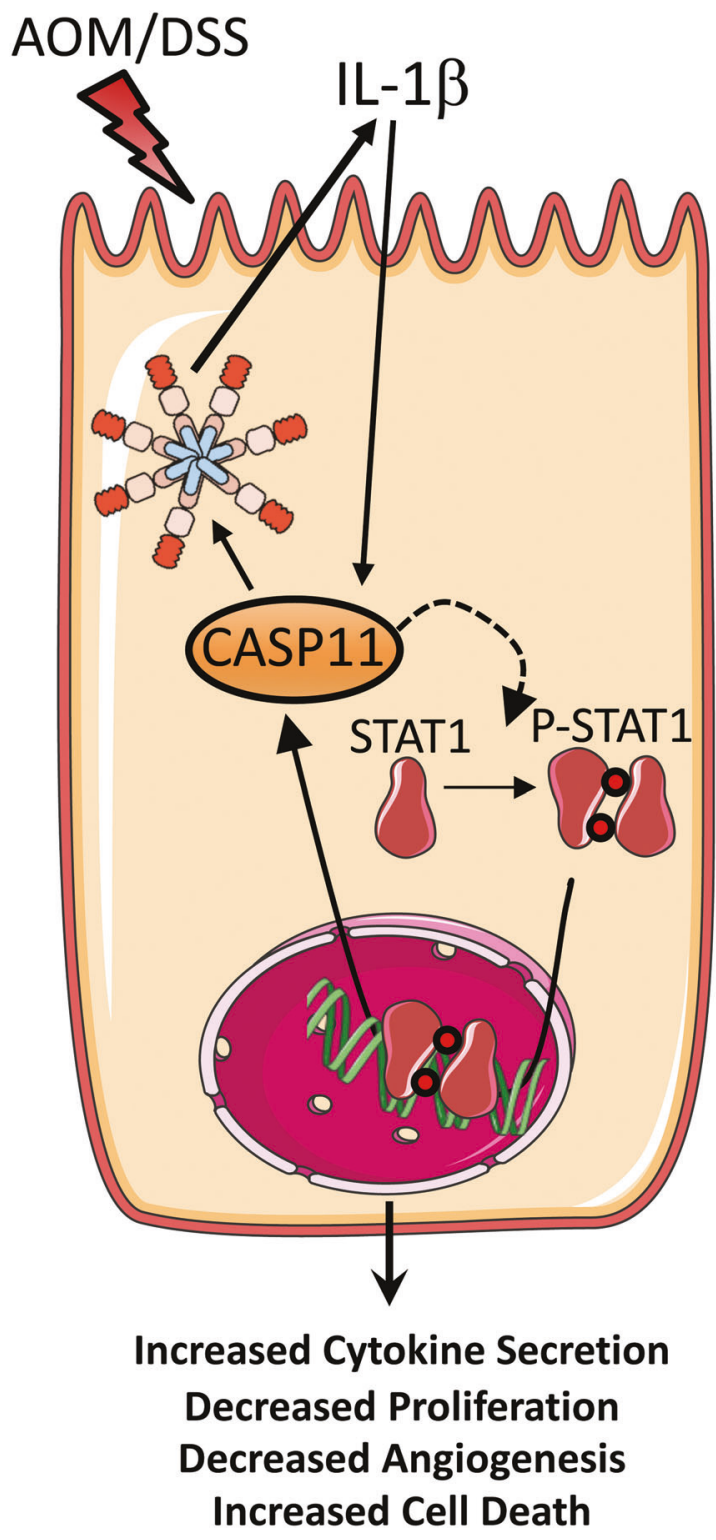

Fig. 8 Graphical representation-the anti-tumorigenic role of caspase11 during CAC. Caspase-11 has dual roles during AOM-DSS-induced colitis-associated carcinogenesis: (i) enhancing IL-1 $\beta$ production during tumorigenesis; and (ii) regulating STAT1 activation in IECs in response to IL-1 $\beta$. The effects of caspase-11 are anti-tumorigenic in this system, causing decreased angiogenesis and increased IEC death

during colorectal carcinogenesis; the ability of IL-1 $\beta$ to induce caspase-11 upregulation; and a requirement for caspase-11 during IL-1 $\beta$-mediated STAT1 activation in IECs.

\section{Discussion}

This study reveals that the anti-tumorigenic effects of STAT1 during CAC are critically dependent upon caspase-

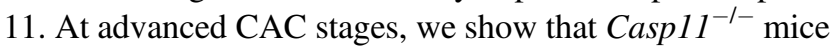
have more aggressive and higher incident tumours than their WT littermates. A previous study on CAC has reported no observable differences between WT and $\operatorname{Caspl1}^{-1-}$ mice [17], however the study performed a shorter trial ( 8.5 week) and observed much fewer adenomas/colon (0.5-1.5 compared with 4-15 in our study), suggesting that differences may have become apparent if the model had been extended.

Consistent with the enhanced tumorigenesis observed in Casp $11^{-/-}$mice, we observe defective STAT1 activation in caspase-11 deficient AOM-DSS-treated mice at both advanced (15 week) and progression (6 week) stages of CAC. Increased angiogenic markers and decreased IEC

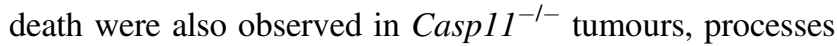
which are usually inhibited by STAT1 [46]. There is significant evidence for the anti-tumorigenic role of STAT1 in both human and murine studies, as STAT1 is believed to be responsible for the anti-proliferative effects of IFN [47]. Studies in patients have suggested a negative correlation between STAT1 and CRC tumorigenesis and tumour cell proliferation [43, 48], and STAT1 has been proposed as a biomarker for good prognosis in CRC [49]. Whether, similar to murine caspase-11, human caspases-4 and -5 regulate STAT1 activity during CRC progression in patients has yet to be determined. In support of our findings, Stat1 ${ }^{-I-}$ mice have increased susceptibility to experimentallyinduced tumours and spontaneously develop mammary adenocarcinomas [50, 51]. Furthermore, a recent study has shown that mice with specific deletion of STAT1 in intestinal epithelial cells (STAT1-DIEC) are more susceptible to AOM-DSS-induced CRC, exhibiting higher grade adenomas than their similarly treated littermate controls [52]. Interestingly, this study found that male STAT1-DIEC mice were significantly more susceptible than their female counterparts, suggesting that, in epithelial compartments, the tumour suppressor function of STAT1 may be influenced by gender [52].

Although we reveal a novel link between the noncanonical inflammasome and STAT1 activity during CAC, components of the canonical inflammasome (NLRP3 and caspase-1), have been previously proposed to mediate their protective effects during CAC via IL-18-mediated IFN $\gamma$ STAT1 signalling [4]. In agreement with this, our study shows that at early stages (3 weeks) of CAC development, reduced IL-18 levels were measured in Casp11 $1^{-1-}$ colon homogenates (compared to their WT littermates), which

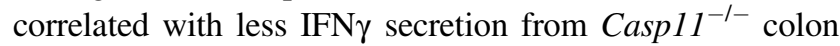
explants. However, at advanced stages of CAC we show that IL-1 $\beta$ levels are more significantly affected than IL-18 levels in $\mathrm{Caspl1}^{-1-}$ colons. These results suggest that caspase-11-mediated IL-1 $\beta$ signalling is protective during experimental CAC. Although high levels of IL-1 $\beta$ have been associated with tumour progression [53], a recent study has suggested that physiological levels of IL-1 are essential for antitumor immunity [54]. For example, IL-1 $\beta$ 
is required for the polarisation of IFN $\gamma$-producing $\mathrm{CD}^{+}$ $\mathrm{T}$ cells; and is also implicated in the immunostimulatory properties of chemotherapy in the generation of anti-tumour $\gamma \delta$ T cells $[55,56]$. In support of our finding, Allen et al. [20] also show impaired IL-1 $\beta$ levels in $A^{-1-}$ and Caspl $^{-1-}$ mice, correlating with increased tumour burden, following 8 weeks AOM-DSS administration.

There are tissue and cell-specific differences in the regulation of caspase-11; while type I IFNs are strongly required for caspase-11 activation in BMDM [15, 57], Ifnar ${ }^{-1-}$ and Trif $^{-1-}$ mice show no impairment in caspase-11 transcription or activation in the murine colon during DSScolitis [12]. Previous data show that IFN $\gamma$-mediated STAT1 signalling is a key requirement for intestinal caspase-11 upregulation and activation in IECs during DSScolitis [12]. The current study demonstrates that caspase-11 is strongly induced in both BMDM and IECs following IL$1 \beta$ stimulation, which has not been previously reported. As IL-1 $\beta$ activates NFkB via IL-1 receptor binding and MyD88 signalling, and caspase- 11 contains $\kappa B$ elements within its promoter region [45], it is not surprising that IL$1 \beta$ is capable of driving caspase- 11 transcription. Therefore, in addition to elucidating a critical role for caspase-11 in IL$1 \beta$ production in established colorectal tumours, our data show that, once initiated, IL- $1 \beta$ signalling drives caspase- 11 upregulation in BMDM, IECs and CT26 murine CRC cells, to further promote its pro-inflammatory effects. Whether IL$1 \beta$ has similar effects on caspase-11 in other cell types has yet to be determined.

This is the first report, to our knowledge, that demonstrates a positive relationship between IL-1 $\beta$ signalling and STAT1 activation, which is regulated by caspase- 11 . We show that caspase-11 is required for IL-1 $\beta$ - and LPS-driven STAT1 activation in IECs, suggesting that caspase-11 facilitates crosstalk between MyD88 and STAT1 signalling pathways at the intestinal barrier, to promote antitumorigenic signalling during inflammation-associated intestinal disease. A protective role for MyD88 during AOM-DSS-induced intestinal carcinogenesis has been previously reported [58]. Impaired induction of IFN-dependent genes were shown in AOM-DSS-treated colons of MyD88 -I- compared to WT mice, which could only be partially explained by defective IL-18 signalling, suggesting that additional TLR/IL-1 signalling pathways, such as IL-1 $\beta$, may contribute to the protective effect of MyD88 during CAC [58]. Although we demonstrate a regulatory role for caspase-11 during LPS and IL-1 $\beta$-mediated STAT1 activation in IECs, LPS-stimulated, but not IL-1 $\beta$ stimulated, primary macrophages from $\mathrm{Casp}_{11^{-1-}}$ mice show impaired STAT1 activation. Thus, similar to the differential regulation of caspase-11 expression in IECs and BMDM [12], the regulatory role of caspase-11 may also differ between innate immune and epithelial cell types. Both LPS- and IL-1 $\beta$ - mediated STAT1 activation in BMDM are type I IFNdependent, however the caspase-11 regulated mechanisms governing STAT1 activation in IECs have yet to be confirmed. Further studies are required to elucidate the mechanistic basis responsible for caspase-11-mediated STAT1 activation. Preliminary data suggest that the levels of negative STAT1 regulators, including SOCS1, are not affected by caspase-11 deficiency (J. Manils, E. Creagh, unpublished). We hypothesise that upregulated caspase-11 is capable of mediating STAT1 activation, either directly via its CARD domain or by the recruitment of another cytosolic molecule. Evidence for this hypothesis comes from previous studies which show a biphasic regulation of STAT1 in response to dsRNA, with an initial IFNdependent phase followed by a STAT1 phosphorylation phase that is IFN-independent $[59,60]$. The CARD domain of RIG-I can induce STAT1 activation independently of Type I IFN, and it has been proposed that a cytosolic CARD-containing molecule may be capable of STAT1 activation [59]. Overexpression studies suggest that IFN responses are amplified by RIG-I, in the absence of viral infection, via direct interaction of the RIG-I CARD region with the STAT1 SH2-transactivation domain, impeding STAT1 dephosphorylation and thereby sustaining its activation [61]. Whether caspase-11 is capable of STAT1 regulation in a manner analogous to that of RIG-I is currently under investigation.

STAT1 has a complex role during tumourigenesis, and it has been proposed to link intestinal inflammation and colon cancer by its involvement in crosstalk between stromal and epithelial cell populations [62]. Our data support the hypothesis that not all IL-1 $\beta$ signalling is involved in tumour promotion, as IL- $1 \beta$ is capable of stimulating caspase-11 expression, which can subsequently mediate STAT1 activation in IECs. This study identifies dual functions for caspase- 11 during CAC - generating IL- $1 \beta$ via the non-canonical inflammasome; and mediating the tumour suppressor activities of STAT1.

\section{Materials and methods}

\section{Mice}

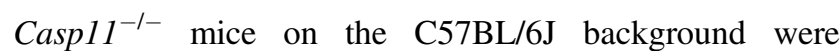
obtained from J. Yuan (Harvard Medical School), and were further backcrossed with C57BL/6J mice for eight generations. Heterozygous breeding pairs were used to generate wild-type (WT) and Casp11 $1^{-/-}$littermates. Experiments were performed with 10-14-week-old female mice bred under specific pathogen-free conditions, under license and the approval of the local animal research ethics committee and the Irish Health Protection Regulatory Agency. The 
Ifnar $^{-1-}$ and Ifn- $\gamma^{-l-}$ mice, used to generate BMDM, were kindly provided by Prof. Ed Lavelle, TCD.

\section{Induction of colitis-associated CRC}

Tumorigenesis in mice was induced by AOM (WAKO Chemicals) injection (intraperitoneally, $12.5 \mathrm{mg} / \mathrm{kg}$ ) on day 0 and 21. On day 7, 28 and 49, 2\% DSS (MP Biomedicals, MW 36 000-50 000) was supplemented in drinking water for 4 days. Animals were weighed and monitored daily for disease activity (weight loss, stool consistency and rectal bleeding (Scoring Table -Supplementary Figure 1a)). On day 105 , mice were humanely killed and colons were removed from the ceco-colonic junction to the rectum. Experiment was performed three times with $n=6$ mice/ coded group (Power $>0.935$; std dev 1.25; $\alpha$ error prob. 0.05: G-power software analysis). Each colon was processed as follows: A $0.5 \mathrm{~cm}$ section was taken from the distal and proximal (adjacent normal control) ends for histology and imaging; the next distal $1 \mathrm{~cm}$ colon tissue section was taken for western blot analysis; and the following $1 \mathrm{~cm}$ (distal-mid colon) section was used for cytokine measurement.

\section{Histology \& Immunostaining}

Following fixation (10\% buffered formalin, $48 \mathrm{~h}$ ), distal colon sections were processed, paraffin embedded, sectioned longitudinally $(5 \mu \mathrm{m})$ and $\mathrm{H} \& \mathrm{E}$ stained. Histological assessment was performed by an experienced pathologist (E. Kay) in blinded fashion. For immunostaining, sections were deparaffinized in Histoclear (National Diagnostics) for $5 \mathrm{~min}$ and progressively rehydrated in decreasing concentrations of ethanol (100, 90 and 70\%, $5 \mathrm{~min}$ ), with final incubation in water. Antigen retrieval for Ki67 (LeicaBiosystems; NCL-Ki67p), phospho-STAT1 (Cell Signalling; 9167), total STAT1 (Cell Signalling; 9172) and E-Cadherin (eBiosciences; 50-3249-80) was carried out by boiling in Tris-EDTA, pH 9.0 (20 min), cooling to RT, $1 \mathrm{~h}$ blocking (PBS, 20\% FBS) before incubation with the primary antibody (1 h, RT). For PCNA (Abcam), antigen retrieval was performed by $10 \mathrm{~min}$ incubation in boiling $0.01 \mathrm{M}$ sodium citrate buffer ( $\mathrm{pH}$ 6.0). Sections were washed (PBST) and incubated with primary antibody $\left(1: 100, \mathrm{o} / \mathrm{n}, 4^{\circ} \mathrm{C}\right)$. Slides were washed and incubated with Alexa Fluor-conjugated secondary antibodies (1:500 dilution, Invitrogen) before mounting (DakoCytomation). Images were obtained using an Olympus BX51 microscope. Quantitative fluorescence intensity of PCNA-positive epithelial cells was analysed in three specifically selected areas of colonic tissue/mouse using Imaris software. For Ki67 and pSTAT1 quantifications, the number of positive cells/10 well-oriented crypts/ mouse were analysed.

\section{TUNEL staining}

Distal colonic tissue sections were analysed by fluorescence microscopy using an in situ cell death detection kit (Roche) according to the manufacturer's protocol. $\times 20$ images of stained tissue sections were taken using the Olympus BX51 microscope. Three random optical fields/colon from each mouse colon were chosen. Within each of the three fields/ mouse, three separate areas (each containing $~ 100$ cells) were enlarged and quantified for TUNEL positivity in control and AOM/DSS-treated animals.

\section{Cytokine measurements}

Sections from tumorigenic distal-mid colon regions were homogenised in lysis buffer (PBS, 1\% NP-40 and Proteaseinhibitor cocktail (Roche)). Colon homogenates were centrifuged $\left(20,000 \times g, 10 \mathrm{~min}, 4{ }^{\circ} \mathrm{C}\right)$ and supernatants normalised using the BCA method (ThermoFisher). For colon explants, colons were opened longitudinally, PBS washed and cut into $3 \mathrm{~cm}$ sections. Each section was cultured in media (DMEM + GlutaMAX, 10\% FBS, 1\% Pen-Strep) for $20 \mathrm{~h}$. Supernatants were analysed for secreted cytokine levels using Biolegend (IL-1 $\beta$, IL-10, IFN- $\gamma$, IL-6 and IL$17 \mathrm{a}$, and IL-1 $\alpha$ ) and R\&D Systems (IL-22) ELISA kits. IL18 levels were determined using IL-18 capture (MBL; DO47-3) and detection (MBL; D048-6) antibodies. IFN- $\beta$ levels were determined using IFN- $\beta$ capture (Santa Cruz; sc57201) and detection (PBL Assay Science; 32400-1) antibodies.

\section{Colon explant stimulation and isolation of colonic epithelial cells}

Colon sections $(3 \mathrm{~cm})$ were untreated or treated with $1 \mu \mathrm{g} /$ $\mathrm{ml}$ LPS (Sigma; E. coli 0111.B4); $20 \mathrm{ng} / \mathrm{ml}$ murine IFN $\gamma$ (Peprotech; 315-05); or $20 \mathrm{ng} / \mathrm{ml}$ murine IL-1 $\beta$ (R\&D; 401ML-005) for $24 \mathrm{~h}$. Sections were agitated $\left(4^{\circ} \mathrm{C}, 2 \mathrm{~h}\right)$ in 0.5 $\mathrm{ml}$ Cell Recovery Solution (BD). Crypts were dislodged by vigorous vortex and separated from the lamina propria fraction by aspiration. Fractions were centrifuged at $(350 \times$ $g, 10 \mathrm{~min})$, pellets lysed (100 $\mu \mathrm{l}$ ice-cold RIPA buffer) and protein concentration was calculated (BCA method) before immunoblotting.

\section{Cell culture and stimulation}

The CT26 cell line was sourced from ATCC. Bone marrow derived macrophages (BMDMs) from WT, Casp11 $1^{-1-}$, IFNAR $^{-1-}$ and IFN- $\gamma^{-1-}$ mice were cultured (DMEM, $10 \%$ FBS, 20\% L929-medium) for 9-11 days, plated at $3 \times 10^{5}$ cells/well in a 24 well plate and cultured o/n. BMDM 
stimulations with LPS, IL-1 $\beta$ and IFN $\gamma$ were performed at the same concentrations used for colon explant stimulations.

\section{Immunoblotting}

Colon tissue was homogenised in RIPA buffer, clarified and normalised using the BCA method. $20 \mu \mathrm{g}$ protein was run on $10-12 \%$ SDS-PAGE gels, transferred to nitrocellulose and probed with primary antibodies against caspase-11 (Sigma; C1354), caspase-1 (Santa Cruz; sc-514), IL-1 $\beta$ (Santa Cruz; sc7884), STAT1 (Cell Signalling; 9172), phospho-STAT1 (Cell Signalling; 7649), STAT3 (Cell Signalling; 8768), phospho-STAT3 (Cell Signalling; 9131), $\beta$-actin (AC-15, Sigma; A3854), Cytokeratin-18 (Santa Cruz; sc-31700) or Vimentin (abcam; EPR3776), followed by incubation with the appropriate HRP-secondary antibody.

\section{Angiogenesis proteome array}

Angiogenic proteins were detected in colon homogenates using an Angiogenesis proteome profiler array (R\&D Systems; ARY015) according to manufacturer's protocol.

\section{Statistical analysis}

Data were analysed using GraphPad Prism 5 software. Unpaired two-tailed Student $t$-tests were used to compare the mean values between two groups. Statistical differences in mean values between more than two experimental groups were determined by two-way analysis of variance (ANOVA) followed by Bonferroni post test. $p$ values $<0.05$ were considered statistically significant.

Acknowledgements We thank the J. Yuan laboratory (Harvard Medical School) for the provision of Caspase-11 ${ }^{-1-}$ mice; and the technical staff of the Comparative Medicine Unit, TCD for their assistance in the backcrossing and breeding of these mice. This study was supported by Enterprise Ireland (CF-2015-0061P). B.F. was supported by a Government of Ireland PRTLI PhD programme (scheme \#201921, award \# 12259). E.F. is an Early Stage Researcher of the EU Horizon 2020 Research and Innovation Programme, TRACT (Marie SklodowskaCurie grant agreement No 721906). S.K. is funded by an SFI Investigator programme grant (12/IP/1400).

\section{Compliance with ethical standards}

Conflict of interest The authors declare that they have no conflict of interest.

Open Access This article is licensed under a Creative Commons Attribution 4.0 International License, which permits use, sharing, adaptation, distribution and reproduction in any medium or format, as long as you give appropriate credit to the original author(s) and the source, provide a link to the Creative Commons license, and indicate if changes were made. The images or other third party material in this article are included in the article's Creative Commons license, unless indicated otherwise in a credit line to the material. If material is not included in the article's Creative Commons license and your intended use is not permitted by statutory regulation or exceeds the permitted use, you will need to obtain permission directly from the copyright holder. To view a copy of this license, visit http://creativecommons. org/licenses/by/4.0/.

\section{References}

1. Jemal A, Bray F, Center MM, Ferlay J, Ward E, Forman D. Global cancer statistics. CA Cancer J Clin. 2011;61:69-90.

2. Neurath MF. Cytokines in inflammatory bowel disease. Nat Rev Immunol. $2014 ; 14: 329-42$.

3. Asquith M, Powrie F. An innately dangerous balancing act: intestinal homeostasis, inflammation, and colitis-associated cancer. J Exp Med. 2010;207:1573-7.

4. Zaki MH, Vogel P, Body-Malapel M, Lamkanfi M, Kanneganti TD. IL-18 production downstream of the Nlrp3 inflammasome confers protection against colorectal tumor formation. J Immunol. 2010;185:4912-20.

5. Mariathasan S, Weiss DS, Newton K, McBride J, O'Rourke K, Roose-Girma M, et al. Cryopyrin activates the inflammasome in response to toxins and ATP. Nature. $2006 ; 440: 228-32$.

6. Martinon F, Petrilli V, Mayor A, Tardivel A, Tschopp J. Goutassociated uric acid crystals activate the NALP3 inflammasome. Nature. 2006;440:237-41.

7. Creagh EM. Caspase crosstalk: integration of apoptotic and innate immune signalling pathways. Trends Immunol. 2014;35:631-40.

8. Kayagaki N, Warming S, Lamkanfi M, Walle LV, Louie S, Dong $\mathrm{J}$, et al. Non-canonical inflammasome activation targets caspase11. Nature. 2011;479:117-21.

9. Kayagaki N, Wong MT, Stowe IB, Ramani SR, Gonzalez LC, Akashi-Takamura S, et al. Noncanonical inflammasome activation by intracellular LPS independent of TLR4. Science. 2013;341:1246-9.

10. Aachoui Y, Leaf IA, Hagar JA, Fontana MF, Campos CG, Zak $\mathrm{DE}$, et al. Caspase- 11 protects against bacteria that escape the vacuole. Science. 2013;339:975-8.

11. Case CL, Kohler LJ, Lima JB, Strowig T, de Zoete MR, Flavell RA, et al. Caspase-11 stimulates rapid flagellin-independent pyroptosis in response to Legionella pneumophila. Proc Natl Acad Sci USA. 2013;110:1851-6.

12. Oficjalska K, Raverdeau M, Aviello G, Wade SC, Hickey A, Sheehan KM, et al. Protective role for caspase-11 during acute experimental murine colitis. J Immunol. 2015;194:1252-60.

13. Gurung P, Malireddi RK, Anand PK, Demon D, Walle LV, Liu Z, et al. TRIF-mediated caspase-11 production integrates TLR4- and Nlrp3 inflammasome-mediated host defense against enteropathogens. J Biol Chem. 2012;287:16955-64.

14. Hur J, Kim SY, Kim H, Cha S, Lee MS, Suk K. Induction of caspase-11 by inflammatory stimuli in rat astrocytes: lipopolysaccharide induction through p38 mitogen-activated protein kinase pathway. FEBS Lett. 2001;507:157-62.

15. Rathinam VA, Vanaja SK, Waggoner L, Sokolovska A, Becker C, Stuart LM, et al. TRIF licenses caspase-11-dependent NLRP3 inflammasome activation by gram-negative bacteria. Cell. 2012;150:606-19.

16. Demon D, Kuchmiy A, Fossoul A, Zhu Q, Kanneganti TD, Lamkanfi M. Caspase-11 is expressed in the colonic mucosa and protects against dextran sodium sulfate-induced colitis. Mucosal Immunol. 2014;7:1504.

17. Williams TM, Leeth RA, Rothschild DE, McDaniel DK, Coutermarsh-Ott SL, Simmons AE, et al. Caspase-11 attenuates 
gastrointestinal inflammation and experimental colitis pathogenesis. Am J Physiol Gastrointest Liver Physiol. 2015;308:G139-50.

18. Dupaul-Chicoine J, Yeretssian G, Doiron K, Bergstrom KSB, McIntire CR, LeBlanc PM, et al. Control of intestinal homeostasis, colitis, and colitis-associated colorectal cancer by the inflammatory caspases. Immunity. 2010;32:367-78.

19. Flood B, Oficjalska K, Laukens D, Fay J, O'Grady A, Caiazza F, et al. Altered expression of caspases- 4 and -5 during inflammatory bowel disease and colorectal cancer: diagnostic and therapeutic potential. Clin Exp Immunol. 2015;181:39-50.

20. Allen IC, TeKippe EM, Woodford RM, Uronis JM, Holl EK, Rogers $\mathrm{AB}$, et al. The NLRP3 inflammasome functions as a negative regulator of tumorigenesis during colitis-associated cancer. J Exp Med. 2010;207:1045-56.

21. Hu B, Elinav E, Huber S, Booth CJ, Strowig T, Jin C, et al. Inflammation-induced tumorigenesis in the colon is regulated by caspase-1 and NLRC4. Proc Natl Acad Sci USA. 2010;107:21635-40.

22. Hu B, Elinav E, Huber S, Strowig T, Hao L, Hafemann A, et al. Microbiota-induced activation of epithelial IL-6 signaling links inflammasome-driven inflammation with transmissible cancer. Proc Natl Acad Sci USA. 2013;110:9862-7.

23. Normand S, Delanoye-Crespin A, Bressenot A, Huot L, Grandjean T, Peyrin-Biroulet L, et al. Nod-like receptor pyrin domaincontaining protein 6 (NLRP6) controls epithelial self-renewal and colorectal carcinogenesis upon injury. Proc Natl Acad Sci USA. 2011;108:9601-6.

24. Chen GY, Liu M, Wang F, Bertin J, Núñez G. A functional role for Nlrp6 in intestinal inflammation and tumorigenesis. J Immunol. 2011;186:7187-94.

25. Williams TM, Leeth RA, Rothschild DE, Coutermarsh-Ott SL, McDaniel DK, Simmons AE, et al. The NLRP1 inflammasome attenuates colitis and colitis-associated tumorigenesis. J Immunol. 2015;194:3369-80.

26. Man SM, Zhu Q, Zhu L, Liu Z, Karki R, Malik A, et al. Critical role for the DNA sensor AIM2 in stem cell proliferation and cancer. Cell. 2015;162:45-58.

27. Wilson JE, Petrucelli AS, Chen L, Koblansky AA, Truax AD, Oyama Y, et al. Inflammasome-independent role of AIM2 in suppressing colon tumorigenesis via DNA-PK and Akt. Nat Med. 2015;21:906-13

28. Elinav E, Strowig T, Kau AL, Henao-Mejia J, Thaiss CA, Booth $\mathrm{CJ}$, et al. NLRP6 inflammasome regulates colonic microbial ecology and risk for colitis. Cell. 2011;145:745-57.

29. Folkman J. Role of angiogenesis in tumor growth and metastasis. Semin Oncol. 2002;29(6Suppl 16):15-8.

30. Lopez-Pastrana J, Ferrer LM, Li YF, Xiong X, Xi H, Cueto R. et al. Inhibition of Caspase-1 activation in endothelial cells improves angiogenesis: a novel therapeutic potential for ischemia. J Biol Chem. 2015;290:17485-94.

31. Wu XL, Lin KJ, Bai AP, Wang WX, Meng XK, Su XL, et al. Osteopontin knockdown suppresses the growth and angiogenesis of colon cancer cells. World J Gastroenterol. 2014;20:10440-8.

32. Fung KY, Tabor B, Buckley MJ, Priebe IK, Purins L, Pompeia C, et al. Blood-based protein biomarker panel for the detection of colorectal cancer. PLoS ONE. 2015;10:e0120425.

33. Peterson JE, Zurakowski D, Italiano JE Jr, Michel LV, Connors S, et al. VEGF, PF4 and PDGF are elevated in platelets of colorectal cancer patients. Angiogenesis. 2012;15:265-73.

34. Biasco G, Paganelli GM, Miglioli M, Brillanti S, Di Febo G, Gizzi $\mathrm{G}$, et al. Rectal cell proliferation and colon cancer risk in ulcerative colitis. Cancer Res. 1990;50:1156-9.

35. Tattoli I, Killackey SA, Foerster EG, Molinaro R, Maisonneuve C, Rahman MA, et al. NLRX1 acts as an epithelial-intrinsic tumor suppressor through the modulation of TNF-mediated proliferation. Cell Rep. 2016;14:2576-86.
36. Barker N. Adult intestinal stem cells: critical drivers of epithelial homeostasis and regeneration. Nat Rev Mol Cell Biol. 2014;15:19-33.

37. Perse M, Cerar A. Dextran sodium sulphate colitis mouse model: traps and tricks. J Biomed Biotechnol. 2012;2012:718617.

38. Hagar JA, Powell DA, Aachoui Y, Ernst RK, Miao EA. Cytoplasmic LPS activates caspase-11: implications in TLR4independent endotoxic shock. Science. 2013;341:1250-3.

39. Kayagaki N, Stowe IB, Lee BL, O'Rourke K, Anderson K, Warming $\mathrm{S}$, et al. Caspase-11 cleaves gasdermin $\mathrm{D}$ for noncanonical inflammasome signalling. Nature. 2015 ;526:666-71.

40. Fink SL, Cookson BT. Caspase-1-dependent pore formation during pyroptosis leads to osmotic lysis of infected host macrophages. Cell Microbiol. 2006;8:1812-25.

41. Boucher D, Monteleone M, Coll RC, Chen KW, Ross CM, Teo $\mathrm{JL}$, et al. Caspase-1 self-cleavage is an intrinsic mechanism to terminate inflammasome activity. J Exp Med. 2018;215:827-40.

42. Gkouskou KK, Ioannou M, Pavlopoulos GA, Georgila K, Siganou A, Nikolaidis G, et al. Apolipoprotein A-I inhibits experimental colitis and colitis-propelled carcinogenesis. Oncogene. 2016;35:2496-505.

43. Nivarthi H, Gordziel C, Themanns M, Kramer N, Eberl M, Rabe B, et al. The ratio of STAT1 to STAT3 expression is a determinant of colorectal cancer growth. Oncotarget. 2016;7:51096-106.

44. Yuasa K, Hijikata T. Distal regulatory element of the STAT1 gene potentially mediates positive feedback control of STAT1 expression. Genes Cells. 2016;21:25-40.

45. Schauvliege R, Vanrobaeys J, Schotte P, Beyaert R. Caspase-11 gene expression in response to lipopolysaccharide and interferon- $\gamma$ requires nuclear factor- $\mathrm{\kappa B}$ and signal transducer and activator of transcription (STAT) 1. J Biol Chem. 2002;277:41624-30.

46. Huang S, Bucana CD, Van Arsdall M, Fidler IJ. Stat1 negatively regulates angiogenesis, tumorigenicity and metastasis of tumor cells. Oncogene. 2002;21:2504-12.

47. Bromberg JF, Horvath CM, Wen Z, Schreiber RD, Darnell JE Jr. Transcriptionally active Stat 1 is required for the antiproliferative effects of both interferon alpha and interferon gamma. Proc Natl Acad Sci USA. 1996;93:7673-8.

48. Ma Y, Chen L, Xie G, Zhou Y, Yue C, Yuan X, et al. Elevated level of interleukin-35 in colorectal cancer induces conversion of $\mathrm{T}$ cells into iTr35 by activating STAT1/STAT3. Oncotarget. 2016;7:73003-15

49. Simpson JA, Al-Attar A, Watson NF, Scholefield JH, Ilyas M, Durrant LG. Intratumoral $\mathrm{T}$ cell infiltration, MHC class I and STAT1 as biomarkers of good prognosis in colorectal cancer. Gut. 2010;59:926-33.

50. Kaplan DH, Shankaran V, Dighe AS, Stockert E, Aguet M, Old $\mathrm{LJ}$, et al. Demonstration of an interferon gamma-dependent tumor surveillance system in immunocompetent mice. Proc Natl Acad Sci USA. 1998;95:7556-61.

51. Chan SR, Vermi W, Luo J, Lucini L, Rickert C, Fowler AM, et al. STAT1-deficient mice spontaneously develop estrogen receptor alpha-positive luminal mammary carcinomas. Breast Cancer Res. 2012;14:R16.

52. Crncec I, Modak M, Gordziel C, Svinka J, Scharf I, Moritsch S, et al. STAT1 is a sex-specific tumor suppressor in colitisassociated colorectal cancer. Mol Oncol. 2018;12:514-28.

53. Dinarello CA. Immunological and inflammatory functions of the interleukin-1 family. Annu Rev Immunol. 2009;27:519-50.

54. Carmi Y, Rinott G, Dotan S, Elkabets M, Rider P, Voronov E, et al. Microenvironment-derived IL-1 and IL-17 interact in the control of lung metastasis. J Immunol. 2011;186:3462-71.

55. Ghiringhelli F, Apetoh L, Tesniere A, Aymeric L, Ma Y, Ortiz C, et al. Activation of the NLRP3 inflammasome in dendritic cells induces IL-1beta-dependent adaptive immunity against tumors. Nat Med. 2009;15:1170-8. 
56. Mattarollo SR, Loi S, Duret H, Ma Y, Zitvogel L, Smyth MJ. Pivotal role of innate and adaptive immunity in anthracycline chemotherapy of established tumors. Cancer Res. 2011;71:480920.

57. Broz P, Monack DM. Noncanonical inflammasomes: caspase-11 activation and effector mechanisms. PLoS Pathog. 2013;9: e1003144.

58. Salcedo R, Worschech A, Cardone M, Jones Y, Gyulai Z, Dai $\mathrm{RM}$, et al. MyD88-mediated signaling prevents development of adenocarcinomas of the colon: role of interleukin 18. J Exp Med. 2010;207:1625-36.

59. Dempoya J, Matsumiya T, Imaizumi T, Hayakari R, Xing F, Yoshida H, et al. Double-stranded RNA induces biphasic STAT1 phosphorylation by both type I interferon (IFN)-dependent and type I IFN-independent pathways. J Virol. 2012;86:12760-9.

60. Bhattacharyya S, Zhao Y, Kay TW, Muglia LJ. Glucocorticoids target suppressor of cytokine signaling 1 (SOCS1) and type 1 interferons to regulate Toll-like receptor-induced STAT1 activation. Proc Natl Acad Sci USA. 2011;108:9554-9.

61. Hou J, Zhou Y, Zheng Y, Fan J, Zhou W, Ng IO, et al. Hepatic RIG-I predicts survival and interferon-alpha therapeutic response in hepatocellular carcinoma. Cancer Cell. 2014;25:49-63.

62. Kaler P, Owusu BY, Augenlicht L, Klampfer L. The role of STAT1 for crosstalk between fibroblasts and colon cancer cells. Front Oncol. 2014;4:88. 